\title{
The Antioxidant tempol delays the MIA-induced osteoarthritis progression in rats via modulation of signaling pathways involving TGF- $\beta 1 /$ SMAD3/NOX4 axis
}

Hagar B. Abo-zalam ( $\nabla$ hagar_belal@o6u.edu.eg )

October 6 University

Rehab F. Abdel-Rahman

National Research Centre

Mohamed F. Abd-Ellah

Al- Azhar University

Rania M. Abdalsalam

Cairo University

Mahmoud M. Khattab

Cairo University

\section{Research Article}

Keywords: Osteoarthritis, monosodium iodoacetate, tempol, NOX4, chondrocytes, autophagy.

Posted Date: May 12th, 2021

DOI: https://doi.org/10.21203/rs.3.rs-499700/v1

License: (c) (i) This work is licensed under a Creative Commons Attribution 4.0 International License.

Read Full License 


\section{Abstract}

Osteoarthritis (OA) is a complex disease characterized by structural, functional, and metabolic deteriorations of the whole joint and periarticular tissues. In the current study, we aimed to investigate the possible effects of tempol on knee OA induced by the chemical chondrotoxic monosodium iodoacetate (MIA) which closely mimics both the pain and structural changes associated with human OA. Rats were administrated oral tempol (100 mg/kg) one week post-MIA injection ( $3 \mathrm{mg} / 50 \mu \mathrm{L}$ saline) at the right knee joints for 21 consecutive days. Tempol improved motor performance and debilitated the MIA-related radiological and histological alterations. Besides, it subsided the knee joint swelling. Tempol decreased the cartilage degradation-related biomarkers as matrix metalloproteinase-13, cartilage oligomeric matrix protein, and fibulin-3. The superoxide dismutase mimetic effect of tempol was accompanied by decreased NADPH oxidase 4 (NOX4), inflammatory mediators, nuclear factor kappa-B (NF-KB), overreleased insulin-like growth factor-1 (IGF-1) and transforming growth factor- $\beta 1$ (TGF- $\beta 1$ ). Tempol decreased the expression of chemotactic cytokine ligand 2 (CCL2), dickkopf-related protein-1 (DKK-1), and protein kinase $C$ (PKC). On the molecular level, tempol reduced the phosphorylated protein levels of p38 mitogen-activated protein kinase (MAPK), phosphoinositide 3-kinase (PI3K), protein kinase B (Akt), and small mother against decapentaplegic 3 homologs (SMAD3). These findings suggest the promising role of tempol in ameliorating MIA-induced knee OA in rats via collateral suppression of the catabolic signaling cascades including TGF-B1/SMAD3/NOX4, NOX4/p38MAPK/NF-KB, and PI3K/Akt/NF-KB and therefore modulation of oxidative stress, catabolic inflammatory cascades, chondrocyte metabolic homeostasis, autophagy, and cell survival.

\section{Introduction}

Osteoarthritis $(\mathrm{OA})$ is a chronic degenerative disease that is characterized by articular cartilage degeneration, subchondral bone sclerosis, and osteophyte formation. OA can arise in different sites, mainly affecting the knees, hips, hands, and spine ${ }^{1,2}$. The chief clinical symptoms include chronic pain, joint instability, stiffness, and radiographic alterations ${ }^{3}$.

The intra-articular injection of monosodium iodoacetate (MIA) is considered a convenient model for knee $\mathrm{OA}^{4}$. MIA directly affects the metabolic balance activity of chondrocytes by disrupting glycolysis as a result of inhibiting the activity of the glyceraldehyde-3-phosphate dehydrogenase (GAPDH) enzyme, which eventually leads to cartilage degeneration ${ }^{5}$. MIA is a fast-progressing chemically induced animal model of OA which is mainly used to observe and evaluate pain behavior as it causes loss of articular cartilage with structural lesions and pain, that resembles human $\mathrm{OA}^{6,7}$.

OA pathogenesis involves activation of signal pathways, regulators, and effectors such as mitogenactivated protein kinases (MAPKs), nuclear factor kappa-light-chain-enhancer of activated B cells (NF-KB), phosphatidylinositol-3-kinase (PI3K), and protein kinase B (Akt) ${ }^{8}$. Inflammatory mediators and reactive oxygen species (ROS) are released in response to OA injury, which further leads to exacerbation of cartilage tissue breakdown via up-regulation of the catalytic degrading matrix metalloproteinases 
(MMPs) ${ }^{9}$. Oxidative stress is elicited by an increased ROS release in response to OA injury. This results in mitochondrial membrane damage, which leads to the release of caspases and pro-apoptotic molecules ${ }^{7}$. Also, ROS mediate the effect of pro-inflammatory cytokines; tumor necrosis factor-alpha (TNF-a), interleukin-1 beta (IL-1 $\beta$ ), and interleukin-6 (IL-6), which prompt the synthesis of MMPs, inflammatory cytokines, and chemokines ${ }^{10}$. This in consequence leads to disturbance in cartilage homeostasis and promotes catabolism via induction of cell death ${ }^{11,12}$.

In January 2020, the American College of Rheumatology (ACR) and the Arthritis Foundation (AF) have been updated the treatment guidelines of knee OA. The treatment options are categorized into: strongly recommended options which include exercise, weight loss, oral and topical nonsteroidal antiinflammatory drugs (NSAIDs), and intra-articular corticosteroids. Besides, the conditionally recommended treatment options include duloxetine, cognitive behavioral therapy, and topical capsaicin. Moreover, the guidelines recommended against the use of glucosamine and chondroitin, hyaluronic acid injections, bisphosphonates, hydroxychloroquine, methotrexate, platelet-rich plasma injections, stem cell injections, tumor necrosis factor (TNF) inhibitors, and interleukin-1 receptor antagonists ${ }^{13}$.

Tempol (4-Hydroxy-2,2,6,6-tetramethylpiperidine-1-oxyl) is a membrane-permeable radical scavenger that possesses an anti-oxidant efficacy which is emphasized through its ability to facilitate the metabolism of a wide range of ROS and reactive nitrogen species (RNS). Tempol reaction with superoxide anion $\left(\mathrm{O}_{2}{ }^{\circ} \otimes\right)$ forms hydrogen peroxides $\left(\mathrm{H}_{2} \mathrm{O}_{2}\right)$ which accounts for its superoxide dismutase (SOD) mimetic action. Besides, tempol prevents the generation of hydroxyl radical $(\cdot \mathrm{OH})$ and hydrogen peroxide $\left(\mathrm{H}_{2} \mathrm{O}_{2}\right)$ by Fenton reaction which is responsible for its catalase-like action. Tempol also improves nitric oxide (NO) bioavailability and catalytically removes the highly reactive peroxynitrite (ONOO囚) species. Accordingly, tempol is considered as a general-purpose redox cycling agent rather than a specific SOD mimetic agent 14. Currently, tempol is subjected to many clinical trials sponsored by Matrix Biomed, Inc. (Los Angeles, CA, USA) such as a phase II clinical trial of treatment of radiation and cisplatin-induced toxicities with tempol, and a clinical trial on a single-patient compassionate use of tempol for the treatment of prostate cancer.

Since the pharmacologic treatments have several limitations in the management of $O A$, researchers have been motivated to find alternative therapeutic agents. To the best of our knowledge, this is the first study to focus on the In-Vivo therapeutic effects and mechanisms of tempol on the knee OA model in rats. In 2000, Cuzzocrea et al., ${ }^{15}$ demonstrated the beneficial effects of tempol on collagen-induced arthritis rodent model as an intracellular radical scavenger which may be useful in the treatment of conditions associated with local or systemic inflammation.

Based on these considerations, the present experimental study aims to explicate the therapeutic outcomes of tempol on the different pathological aspects of MIA-induced knee OA, including; a] functional evaluation of motor balance and pain, b] structural alterations (using physical assessment of knee joint edema, X-ray imaging, and histopathology), and c] knee joint degradation-related. In addition, 
we aimed to investigate the potential mechanisms and signaling pathways of tempol in ameliorating MIA-induced OA in rats involving; a] oxidative phosphorylation status, b] oxidative stress biomarkers, c] inflammatory and pain mediators, d] OA-overexpressed growth, e] intracellular signaling proteins (PKC, SMAD3, p38MAPK, PI3K, and Akt), and f] autophagy-related protein (beclin-1).

\section{Materials And Methods}

\subsection{Ethical statement}

All procedures for handling, use, and euthanasia of animals were reviewed and approved by the Ethical Committee for Animal Experimentation of the Faculty of Pharmacy, Cairo University, with the permit number PT 2389, and were performed following the guides for the Care and Use of Laboratory Animals published by the US National Institutes of Health ( $8^{\text {th }}$ edition, NIH Publication, 2011).

\subsection{Experimental animals}

A total of 60 adult male Wistar albino rats (180-200 g; 8-10 weeks old) were obtained from the Animal House Colony at the National Research Centre (NRC, Giza, Egypt). Rats were randomly assigned to four groups $\left(4-5 /\right.$ cage) in a room with a controlled temperature $\left(22^{\circ} \mathrm{C} \pm 1\right)$ on a $12 / 12$ light-dark cycle. All animals were provided with standard rat chow with free access to water. Animals were adapted to the same laboratory environment for 2 weeks before the experimentation. Adequate non-pharmacological measures were taken to minimize animal pain or discomfort according to the National Centre for the Replacement, Refinement and Reduction of Animals in Research (NC3Rs) strategy and Animal Research: Reporting of In-vivo Experiments (ARRIVE) guidelines.

\subsection{Induction of Osteoarthritis}

Single unilateral intra-articular injection of monosodium iodoacetate (MIA) was used to induce knee osteoarthritis in rats. It was purchased from Sigma-Aldrich Chemical Co. (Lot\# SLBZ7569, St. Louis, USA). On day zero, rats were anesthetized with $4 \%$ isoflurane inhalation. Then MIA ( $3 \mathrm{mg}$ dissolved in 50 $\mu \mathrm{L}$ of sterile saline) was injected through the patellar ligament into the joint space of the right knee using a sterile $100-U$ insulin syringe ${ }^{11,12,16-19}$. The sham groups were injected with $50 \mu \mathrm{L}$ physiologic saline in their right knees instead of MIA ${ }^{20}$.

\subsection{Experimental design}

The experimental protocol is summarized in (Figure. 1). Sixty adult male rats were randomly allocated into four groups ( $N=15$ /group). In the first sham group, the animals were subjected to a single intraarticular injection of normal saline in their right knee joints on day 0 and then administered a daily oral dose of the vehicle ( $1 \mathrm{ml}$ of distilled water), one week after saline injection for 21 days. In the second group (sham+tempol), rats were exposed to intra-articular injection of normal saline in their right knee joints on day 0 and then administered tempol $(100 \mathrm{mg} / \mathrm{kg} / \mathrm{day})$ by oral gavage starting from the $7^{\text {th }}$ day 
of the experiment for 21 consecutive days. In the third MIA-induced OA group, OA was induced by a single unilateral intra-articular injection of MIA on day 0 in a dose of $3 \mathrm{mg}$ dissolved in $50 \mu \mathrm{L}$ saline. In the fourth MIA+tempol group, osteoarthritic rats were administered tempol (Sigma-Aldrich Chemical Co., St. Louis, USA) starting from the $7^{\text {th }}$ day of the experiment in a dose of $(100 \mathrm{mg} / \mathrm{kg} /$ day) by oral gavage for 21 consecutive days. Tempol was freshly prepared and daily dissolved in pure distilled water in a concentration of $0.4 \mathrm{~g} / 20 \mathrm{ml}$. The choice of tempol dose was on a pilot trial guided by previously published studies to figure out the appropriate method of administration ${ }^{21-24}$ (Supplementary file 1). In this study, we did not compare with a pharmacological control since the existing approved pharmacological treatments for OA are limited for the relief of OA symptoms (e.g. NSAIDs and corticosteroids), and there is a lack of licensed disease-modifying osteoarthritis drugs (DMOADs) that could slow the narrowing of joint space and provide symptomatic relief.

\subsection{Assessment of knee joint edema}

The diameter (in $\mathrm{mm}$ ) of the right knee of each rat in the studied groups ( $\mathrm{N}=8 / \mathrm{group}$ ) was measured using a calibrated digital caliper (SL-1112, INSIZE Co., USA), at the corresponding days $0,1,3,10$, 17, and $24^{25}$.

\subsection{Evaluation of motor performance using accelerating rotarod test}

Motor stability, coordination, and pain were evaluated using an accelerating rotarod apparatus (Model 7750, Ugo Basile, Italy). Each rat was trained through 3 different sessions for three consecutive days (one session/day) just before the experimentation. Hence, rats were randomly placed on the rotating rod forcing them to walk continuously with increasing speed (from 4 to $40 \mathrm{rpm}$ ) for 5 min (300 s) ${ }^{26}$. Throughout the experiment, rats were tested for their persistence on the accelerating rotarod according to the specific schedule of the different groups on study days $1,7,14,21$, and 28 . The duration that each rat was able to maintain on the rod was recorded as latency time to fall in seconds ( $N=8 /$ group) 27,28 .

\subsection{Radiographical examination}

On day 28 , rats were anesthetized by ketamine/xylazine $(100 / 10 \mathrm{mg} / \mathrm{kg}$, i.p.). Afterward, the rat's right knee was flexed in the anterior to posterior position (AP view, angle $10^{\circ}$ ), and lateral position (angle $30^{\circ}$ ) with an appropriate focal length to ensure clear X-ray imaging ${ }^{29}$. The X-ray films were captured by $50 \mathrm{~mA}$ mobile X-ray camera apparatus (Model F50-100II 50mA, Perlong Medical Equipment Co., China). All X-ray images were blindly examined by two investigators.

\subsection{Blood sampling}

At the end of the experiment on day 28 , animals were killed with an overdose of pentobarbital sodium (200 mg/kg, i.p.) euthanasia solution. Afterward, blood samples were separated and allowed to coagulate. Therefore, samples were introduced into a cooling centrifuge (2-16KL, Sigma Laborzentrifugen GmgH, Germany) at 10,000×g for 15 min to separate serum which used to assess C-telopeptide of type II 
collagen (CTX-II; MBS700687, MyBioSource, USA), cartilage oligomeric matrix protein (COMP; MBS2512987, MyBioSource, USA), interleukin-1 beta (IL-1 $\beta$; EK0393, BosterBio, CA, USA), and insulin-like growth factor-1 (IGF-1; CSB-E04582r, Cusabio Biotech Co., China) using enzyme-linked immunosorbent assay (ELISA) according to manufacturer's procedures.

\subsection{Tissue sampling}

Immediately after blood collection, the right knee joints were separated and divided into 3 sets. The tissue samples of the first set ( $\mathrm{N}=6 /$ group) were reserved for the colorimetric assay of superoxide dismutase (SOD) activity according to the method of Nishikimi et al., ${ }^{30}$, and ELISA assay of the autophagy-related biomarker, beclin-1, bone alkaline phosphatase (Bone ALP), cytochrome c oxidase (complex IV) (CcO), interleukin-6 (IL-6), matrix metalloproteinase-13 (MMP-13), NADPH oxidase 4 (NOX4), nuclear factorkappa $\mathrm{B}_{\mathrm{p} 65}$ subunit (NF-KB), and transforming growth factor- $\beta 1$ (TGF- $\beta 1$ ). For the second tissue set, the tissue samples ( $\mathrm{N}=6 /$ group) were utilized for biochemical analysis of the expression of chemotactic cytokine ligand 2 (CCL2), dickkopf-related protein-1 (DKK-1), discoidin domain receptor 2 (DDR2), fibulin-3 (fib-3), and protein kinase $\mathrm{C}$ (PKC) using real-time polymerase chain reaction (RT-PCR) analysis, and biochemical analysis of the phosphorylated protein levels of p38 mitogen-activated protein kinase (p38MAPK), phosphoinositide 3-kinase $\mathrm{p} 85 / \mathrm{p} 55(\mathrm{PI} 3 \mathrm{~K})$, protein kinase $\mathrm{B}$ (Akt), and small mother against decapentaplegic 3 homologs (SMAD3). For the last tissue set, the tissue samples ( $N=3 /$ group) were fixed in $10 \%$ formalin for histopathological evaluation.

\subsection{Biochemical ELISA measurements}

Knee joint tissues were minced, placed in a round bottom microtube, snap-frozen in liquid nitrogen, and homogenized with an electric homogenizer in five volumes of extraction buffer (100 mM Tris, pH 7.4, 150 $\mathrm{mM} \mathrm{NaCl}, 1 \mathrm{mM}$ EGTA, $1 \mathrm{mM}$ EDTA, $1 \%$ Triton X-100, and 0.5\% sodium deoxycholate). The tissue lysates were placed on a shaker at $4^{\circ} \mathrm{C}$ for $1 \mathrm{~h}$ and centrifuged at $10,000 \times \mathrm{g}$ for $5 \mathrm{~min}$. The supernatants were stored at $-80^{\circ} \mathrm{C}$ for further analysis of beclin-1 (MBS901662, MyBioSource, USA), Bone ALP (CSBE11865r, Cusabio Biotech Co., China), CcO (CSB-E14281r, Cusabio Biotech Co., China), IL-6 (R6000B, R\&D systems, China), MMP-13 (CSB-E07412r, Cusabio Biotech Co., China), NOX4 (LS-F5720, LSBio, USA), NF$\mathrm{KB}_{\mathrm{p} 65}$ (MBS722386, MyBioSource, USA), and TGF- $\beta 1$ (MBS175833, MyBioSource, USA) using rat specific ELISA kits according to the manufacturer's instructions.

\subsection{Quantitative real-time PCR (qRT-PCR) measurements for gene expression}

The messenger RNA (mRNA) expression levels of CCL2, DKK-1, DDR2, fibulin-3, and PKC were analyzed by quantitative real-time PCR. About $30 \mathrm{mg}$ of knee joint tissue was homogenized in RNA lysis solution supplied by RNeasy mini kit (QIAGEN, Maryland, CA, USA) and centrifuged at 10,000×g for $10 \mathrm{~min}$, the supernatant was used for RNA extraction according to the manufacturer's protocol. The concentrations of the isolated RNA were obtained using ultraviolet spectrophotometry, and RNA purity was assessed based on the A260/A280 absorption ratio. Then, RNA was reverse-transcribed into cDNA as described in the manufacturer's using High Capacity cDNA reverse transcription kit (Applied Biosystems, Foster City, CA, 
USA). Real-time PCR was performed with a PCR mixture containing $1 \mu \mathrm{mol} / \mathrm{I}$ of each primer and SYBR Green Master Mix Applied Biosystems, Foster City, CA, USA) using StepOne ${ }^{T M}$ PCR system (version 3.1, Applied Biosystems, Foster City, CA, USA). The sequences of the primers used are listed in (Table 1). All primer sets had a calculated annealing temperature of $60^{\circ} \mathrm{C}$. Amplification conditions were: $95^{\circ} \mathrm{C}$ for 10 $\mathrm{min}$, and then 40 cycles of denaturation for $15 \mathrm{~s}$ and annealing/extension at $60^{\circ} \mathrm{C}$ for $10 \mathrm{~min}$. All values were normalized to the $\beta$-actin which was used as the endogenous control (reference gene). The relative expression of the target genes was obtained using the comparative threshold cycle $C_{T}\left(\Delta \Delta C_{T}\right)$ method. The relative expression was calculated from the $2^{-\Delta \Delta C T}$ formula ${ }^{31}$.

\subsection{Western blot analysis}

Frozen knee joints were weighed and broken into pieces on dry ice. Then were homogenized in Radio Immunoprecipitation Assay (RIPA) lysis buffer (50 mM Tris- $\mathrm{HCl}, 150 \mathrm{mM} \mathrm{NaCl}, 1 \%$ Triton X-100, and 1\% deoxycholate) supplemented with $15 \mathrm{mM}$ sodium fluoride, $1 \mathrm{mM}$ sodium vanadate, $2 \mathrm{mM}$ sodium pyrophosphate, $1 \mathrm{mM}$ sodium glycerophosphate, $2 \mathrm{mM}$ imidazole, $100 \mathrm{mg} / \mathrm{ml}$ phenylmethylsulfonyl fluoride and proteinase inhibitor cocktail (Thermo Fisher Scientific Inc., USA), and sonicated in the lysis buffer, cleared by centrifugation at $12,000 \times \mathrm{g}$ for $10 \mathrm{~min}$ at $4^{\circ} \mathrm{C}$. The protein was quantified using Bradford Protein Assay Kit (Bio Basic Inc. Markham Ontario, L3R 8T4, Canada) ${ }^{32}$ and kept at $-80^{\circ} \mathrm{C}$ until use. Briefly, $50 \mu \mathrm{g}$ total protein samples were resolved on sodium dodecyl sulfate (SDS)-polyacrylamide gel electrophoresis (PAGE) gels which were prepared using TGX Stain-Free ${ }^{T M}$ FastCast $^{\text {TM }}$ Acrylamide Kit (BioRad Laboratories, Inc., Hercules, CA, USA), and then transferred to polyvinylidene difluoride (PVDF) membranes (Sigma-Aldrich Chemical Co., St. Louis, MO, USA), using a semi-dry transfer apparatus BioRad Trans-Blot Turbo Apparatus (Bio-Rad Laboratories Inc., Hercules, CA, USA). The membranes were blocked with $3 \%$ bovine serum albumin (BSA) in Tris-buffered saline with Tween-20 (TBST) at $4^{\circ} \mathrm{C}$ for 1 $\mathrm{hr}$. The membranes were then incubated overnight on a roller shaker at $4{ }^{\circ} \mathrm{C}$ with the following primary rabbit/IgG polyclonal antibodies (Thermo Fisher Scientific Inc., USA): anti-SMAD3 (1:1000; PA5-17378), anti-phospho-(p)-SMAD3 (1:1000, Cat\# PA5-104942), anti-p38 MAPK (1:200, Cat\# OPA1-10080), antiphospho-(p)-p38 MAPK (1:1000, Cat\# 44-684G), anti-PI3K p85/p55 (1:1000, Cat\# PA5-99518), antiphospho-(p)-PI3K p85/p55 (1:1000, Cat\# PA5-99367), anti-Akt (1:2000, Cat\# 100-401-401), anti-phospho(p)-Akt (1:2000, Cat\# 600-401-268), and anti- $\beta$-actin (1:2000, Cat\# PA1-46296). After washing in TBST, the membranes were probed with HRP-conjugated goat anti-rabbit IgG secondary antibody (1:1000, Cat\# HAF008, Novus Biologicals, LLC, USA) at $4^{\circ} \mathrm{C}$ for $1 \mathrm{hr}$. Finally, the protein bands were developed using a Clarity ${ }^{\mathrm{TM}}$ Western ECL chemiluminescent substrate (Cat\# 170-5060, Bio-Rad Laboratories Inc., Hercules,

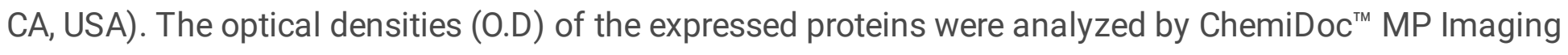
System using Image Lab ${ }^{\text {TM }}$ Software (version 5.1, Bio-Rad Laboratories Inc., Hercules, CA, USA). The O.D of the subsequently mentioned results was normalized to $\beta$-actin.

\subsection{Histopathological Examinations}

Autopsy samples were taken from the rats' right knee joint of different groups ( $\mathrm{N}=3 / \mathrm{group})$ and fixed in $10 \%$ neutral buffered formalin for $48 \mathrm{hr}$. Followed by decalcification by using Cal-Ex ${ }^{\mathrm{TM}}$ II 
Fixative/Decalcifier (Fisher Chemical ${ }^{\text {TM }}$ Scientific, USA) for 20 days. Joints were trimmed and sectioned at the mid-sagittal point then serial step sections were obtained and examined at different levels.

Subsequently, samples were processed using serial dilutions of ethanol and cleared in xylene followed by infiltration and embedding in paraplast tissue embedding media. Tissue sections ( $5 \mu \mathrm{m}$ thickness) were made by rotatory microtome and mounted on glass slides for hematoxylin and eosin (H\&E) staining for general histological examination of joint samples, and also for histochemical assessment by alcian blue stain ( $A B ; \mathrm{pH} 2.5)$ for assessment of cartilage extracellular matrix and proteoglycans reactivity. Nuclear fast red was used as a standard counterstain for alcian blue staining. All guidelines for sample fixation, processing, and staining were done according to Culling et al., ${ }^{33}$. Morphological assessment of tibiofemoral articular cartilage was conducted according to the modified Mankin scoring system ${ }^{34,35}$ with 0 indicating normal cartilage and 13 indicating the maximal score of osteoarthritis.

\subsection{Statistical analysis}

Statistical comparisons between different means of the biochemical parameters were carried out using one-way analysis of variance (ANOVA) followed by Tukey's multiple comparison test. Furthermore, knee joint diameter and rotarod latency time were tabulated and analyzed statistically using repeated measures ANOVA test for factors time and group, followed by the Bonferroni post-hoc test. Statistical analysis was performed using GraphPad Prism (version 5.0, GraphPad Software, Inc., San Diego, USA). All data points are presented as the means \pm SD. A difference was considered to be statistically significant at $P<0.05$.

\section{Results}

\subsection{Effect of tempol on knee joint degradation-related biomarkers}

MIA injection resulted in extracellular matrix (ECM) degradation associated with over-release of MMP-13 contents by 2.7 folds $(P<0.001)$ as compared to the sham group (Fig. $2 a)$. On the same line, we also analyzed DDR2 by qRT-PCR for its role in MMP-13 expression. DDR2 mRNA expression level was increased in the MIA-OA group by 7.0 folds $(P<0.001)$ when compared to the sham group $(\mathrm{Fig} .2 \mathrm{~b})$. Unsurprisingly, MIA further increased mRNA expression of fibulin-3 ( 6.2 folds, $P<0.001)$ and bone ALP activity ( 6.2 folds, $P<0.001)$ when compared to the sham group (Fig. $2 c \& d)$ which sensibly connected to articular damage indication. Conversely, post-treatment with tempol reversed these elevations by $(2.2$ folds, $P<0.001$ ) MMP-13, (2.2 folds, $P<0.001$ ) DDR2, (3.1 folds, $P<0.001)$ fibulin-3, and ( 2.5 folds, $\mathrm{P}<0.001$ ) bone ALP, compared to the MIA-induced OA group (Fig. 2a-d).

In the same context, MIA-OA rats exhibited a spike increase in the serum levels of CTX-II, and COMP by 15.0 and 4.4 folds, respectively, as compared to the sham group $(P<0.001)$. On the other hand, the tempol post-treated group showed marked reductions in serum CTX-II (4.5 folds, $\mathrm{P}<0.001)$ and serum COMP (2.5 folds, $P<0.001)$ relative to the MIA group (Fig. 2e\&f).

\subsection{Effect of tempol on mitochondrial complex IV oxidase and oxidative stress biomarkers}


MIA disrupted the knee joint activity of $\mathrm{CcO}$ (complex IV or the terminal enzyme of the respiratory chain of the mitochondria) by $39.2 \%$ ( $\mathrm{P}<0.001)$ as compared to the sham group (Fig. 3a). Besides, SOD was analyzed calorimetrically and its activity was diminished in the diseased knee joints by MIA by $79.9 \%$ $(P<0.001)$ as compared to the sham group (Fig. 3b). In contrast, NOX4 which constitutively produces $\mathrm{H} 2 \mathrm{O} 2$ was significantly elevated in the MIA-induced OA group by four folds $(P<0.001)$ compared to the sham group (Fig. 3c). However, tempol post-treatment increased $\mathrm{CcO}(53.3 \%)(P<0.001)$ and SOD $(218.7 \%)(P<0.001)$ significantly, along with a noticeable decline in NOX4 by $52.0 \%(P<0.001)$ as relative to the MIA-OA group (Fig. 3a-c).

\subsection{Effect of tempol on knee joint swelling}

Figure (4a) demonstrated the physical assessment of the knee joint edema by the digital caliper. Symptoms of the joint swelling were observed after the intra-articular injection of MIA especially at the first 10 days of the experiment schedule, where, the diameter of the diseased right knee joints showed marked increases on day 1 (73.05\%, $\mathrm{P}<0.001)$, day $3(62.04 \%, \mathrm{P}<0.001)$, and day $10(41.38 \%, \mathrm{P}<0.001)$ as compared to the sham group. In contrast, relief in joint swelling by $(20.88 \%, P<0.001)$ was observed starting from day 10 of the experiment (the 4th day of treatment) compared to the MIA-OA group. On the other hand, tempol post-treated rats exhibited normal knee diameter measures at days 17 and 24, relative to sham groups.

\subsection{Effect of tempol on rotarod performance test}

The accelerating rotarod was used for the functional assessment of motor balance and pain as presented in (Fig. 4b). Progressive motor dysfunction was observed after MIA insult, where, MIA-OA rats displayed considerable reductions in the latency time to fall at the corresponding day 1 (1.98 folds, $P<0.001)$, day 7 ( 2.02 folds, $P<0.001)$, day 14 ( 2.36 folds, $P<0.001$ ), day 21 ( 3.43 folds, $P<0.001$ ), and day 28 ( 3.16 folds, $P<0.001$ ) when compared to the sham group. Similar findings were exhibited by tempoltreated rats at days 1,7 , and 14 as related to the MIA-OA group. On the contrary, tempol post-treatment revealed pronounced improvements in the motor coordination through significant increases of latency time to fall at day 21 ( 2.55 folds, $P<0.001)$, and day 28 ( 2.86 folds, $P<0.001)$ as compared to the MIA-OA group.

\subsection{Effect of tempol on inflammatory and pain mediators}

The MIA-aggravated inflammatory state was demonstrated by marked increments in the serum level of the pro-inflammatory IL-1 $\beta$ ( 2.6 folds, $P<0.001$ ), prominent escalation of knee joint contents of IL-6 (3.4 folds, $P<0.001)$ and NF-kBp65 (3.6 folds, $P<0.001)$. Besides, significant rises of the mRNA expression levels of the chemokine CCL2 (6.3 folds, $\mathrm{P}<0.001)$ were noticed in the MIA-induced OA group compared to the sham group (Fig. 4c-f). In contrast, post-administration of tempol attenuated these elevations, resulting in 57.6\% (serum IL-1 $\beta, P<0.001$ ), 46.0\% (IL-6, $P<0.001$ ), 2.3 folds (NF-kBp65, $P<0.001$ ), and $55.5 \%(C C L 2, P<0.001)$ lower levels in the tempol treated group than in the MIA-induced OA group (Fig. 4Cf). 


\subsection{Effect of tempol on DKK-1 and beclin-1}

Elevated mRNA expression levels of DKK-1 (4.9 folds, $\mathrm{P}<0.001$ ) were noticed in the MIA-induced OA group corresponding to the sham group, but conversely, the mRNA expression level of DKK-1 was reduced by $67.5 \%$ in the tempol-treated group as compared to MIA-OA ones $(P<0.001)$ (Fig. $4 \mathrm{~g})$. On the other hand, the autophagy-related biomarker, beclin-1 tissue contents were decreased $(70.9 \%, P<0.001)$ in the MIA-OA group as compared to the sham group, and in contrast, tempol post-treatment increased beclin- 1 contents $(28.9 \%, \mathrm{P}<0.001)$ in the osteoarthritic rats (Fig. $4 \mathrm{~h})$.

\subsection{Effect of tempol on growth factors and PKC expression}

A metabolic shift has occurred following MIA injection toward catabolic cascade which is characterized by the increased production and activation of different anabolic and catabolic factors that act as mediators of cartilage loss sequence as shown in (Figure 5). This may be considered as a compensative mechanism from chondrocyte to counteract the MIA destructive effect, where significant increments in tissue TGF- $\beta 1$ ( 3.3 folds, $P<0.001$ ), serum IGF-1 ( 8.3 folds, $P<0.001$ ), and mRNA expression levels of PKC (6.2 folds, $P<0.001$ ) were pronounced in MIA-OA group when compared to the sham group (Fig. $5 a-C)$. On the contrary, these effects were mitigated by tempol post-treatment by reducing TGF- $\beta 1$ ( 2.1 folds, $P<0.001)$, and PKC $(51.8 \%, P<0.001)$ as well as restoring serum IGF-1 to its normal value, relative to the MIA-induced OA group (Fig. 5a-C).

\subsection{Effect of tempol on p-SMAD3, p-p38MAPK, p-PI3K, and p-Akt signaling proteins}

Furthermore, MIA insult inappropriately induced marked elevations of the western blot analyzed phosphorylated proteins; p-SMAD3 (7.2 folds, $P<0.001$ ), p-p38MAPK (5.2 folds, $P<0.001)$, p-PI3K (6.7 folds, $P<0.001)$, and $p-A k t(5.3$ folds, $P<0.001)$ as compared to the sham group. Conversely, tempol posttreatment effectively suppressed the phosphorylated protein levels of p-SMAD3 (60.9\%, $P<0.001)$, of $p$ p38MAPK (51.8\%, P<0.01), p-PI3K (2.5 folds, $\mathrm{P}<0.001)$, and $\mathrm{p}$-Akt $(2.0$ folds, $\mathrm{P}<0.001)$ relative to the MIAinduced OA group (Fig. 5d-g).

\subsection{Effect of tempol on the radiographic changes}

The experiment was conducted for X-ray evaluation on day 28. The X-ray images of the right knee joints of the sham groups (Fig. 6a\&b) showed normal joint structure, normal joint spaces, normal radio-density, and normal surfaces of the femoral condyles and proximal tibia. On the other hand, the osteoarthritic changes of the MIA-induced group (Fig. $6 \mathrm{c}$ ) revealed increased joint opacity corresponding to joint effusion and osteophytes formation. Besides, rough surfaces of the femoral condyles and proximal tibia were also observed corresponding to the articular surfaces lysis. However, the radiographs related to tempol post-treated rats showed almost normal joint space with little lysis of the femoral articular surface, and few osteophytes (Fig. 6d).

\subsection{Effect of tempol on the histopathological alterations}


The sham groups showed normal histological structures of articular cartilage layers with apparent intact isogenous chondrocytes. Regular smooth articular surfaces and intact synovial membrane were also observed. Besides, well-organized cartilaginous matrices were recorded with strong high density and reactivity of proteoglycans to alcian blue stain (Fig. 7a\&b). Severe degenerative and necrotic changes with significant loss of chondrocytes were found in MIA-induced OA photomicrographs accompanied by focal erosions and fissures of articular cartilage superficial zones (Fig. 7c1), in addition to occasional subchondral extravasation of blood (Fig. 7c2), edema with inflammatory cell infiltrations of synovial membranes (Fig. 7c3). Significant loss of proteoglycans reactivity to alcian blue was recorded all over articular surfaces in the MIA-OA group (Fig. 7c4). On the other hand, photomicrographs of the tempol post-treated group demonstrated distinguished improvements in the chondrocytes (Fig. 7d1\&2) with mild inflammatory cells synovial membrane infiltrates (Fig. 7d3). Post-treatment with tempol was able to retain the higher reactivity of proteoglycan (Fig. 7D4). Modified Mankin score was recorded in the histogram panel (Fig. 7e). MIA-induced OA group attained the maximal mean of the modified Mankin score of $(10 \pm 1.2, P<0.001)$ as compared to the sham group. However, the mean of modified Mankin score of tempol post-treated group was found to be $(2.5 \pm 0.5, \mathrm{P}<0.001)$ relative to the MIA-OA group.

\section{Discussion}

The present study demonstrates the potential role of tempol as a membrane-permeable radical scavenger in attenuating the related physical, structural, functional, and biochemical deleterious alterations of the knee osteoarthritis rodent model (Figure 8). Accordingly, OA was induced by a single unilateral intraarticular injection of monosodium iodoacetate (MIA) in the rat's right knee joint. MIA is a simple induced and non-invasive experimental model for $\mathrm{OA}^{36}$. MIA prompts chondrocyte death with functional, radiological, histological, and biochemical alterations that resemble human $O A$ in the early phases $17,37,38$. In the current investigation, the degenerative effects of MIA on cartilage are demonstrated biochemically by predominant elevations of MMP-13 contents and the cell surface DDR2 expression levels in the MIA-OA group, our results are consistent with previous studies ${ }^{25,26}$.

A variety of MMPs are produced by OA chondrocytes, among which is MMP-13 or collagenase- 3 which contributes mostly to degrade collagen type II besides proteoglycans and other ECM components. Accordingly, MMP-13 is considered the major catabolic effector in $\mathrm{OA}^{39}$. The proteoglycans depletion consequently augments the exposure and interaction of collagen type II with DDR2 resulting in its activation and further upregulation of MMP-13 ${ }^{40}$. Consequently, the chondrocytes undergo hypertrophy and lose their ability to form new cartilage matrix constituents. Hereafter, the subchondral bone undergoes abnormal remodeling, leading to osteophytes formation as an attempt to correct the resulted joint deformity ${ }^{41}$.

In the present investigation, the destructive effect of MIA on the cartilage is presented by MIA-related structural alterations, where, the radiographic findings reveal joint opacity and osteophytes formation associated with rough surfaces of the femoral condyles and proximal tibia. Besides, the 
histopathological findings show severe degeneration in the chondrocytes and severe loss of proteoglycans reactivity in response to alcian blue staining. Similar findings are mentioned before $6,17,26$.

In addition, further biochemical investigations of cartilage turnover biomarkers indicated elevations in fibulin-3 expression, bone ALP activity, and serum levels of CTX-II and COMP, which may be subordinate to MIA-induced MMP13 upregulation. Fibulin-3 is a member of the ECM proteins that provides organization and stabilization to ECM structure ${ }^{42}$. Bone ALP is expressed in injured cartilage tissues on the cell surface and within matrix vesicles ${ }^{43}$. Furthermore, CTX-II is a product of collagen type II denaturation, Likewise, COMP, or called thrombospondin- 5 is one of the non-collagen ECM components 43 . They are prognostic biomarkers for cartilage breakdown as their levels are highly increased in serum and synovial fluid following MIA insult ${ }^{44,45}$.

Contrariwise, the post-treatment of tempol repressed all MIA-induced elevations of knee joint degradationrelated biomarkers. Moreover, tempol showed a little lysis of the femoral articular surface and few osteophytes in the related radiographs, and also retained the higher proteoglycan reactivity in response to alcian blue staining. Thus, these outcomes suggest the potential role of tempol in reversing the MIAdestructive effect on the cartilage and further highlight the anti-osteoarthritic efficacy of tempol.

MIA disrupts glycolysis and aerobic cellular respiration via inhibition of glyceraldehyde-3-phosphate dehydrogenase $(G A P D H)^{6,46}$. As a consequence, MIA instigated oxidative phosphorylation disruption, ROS elevation, the release of cytochrome $c$ from the mitochondrion, activation of caspase- 3 , and finally provoking chondrocytes apoptosis in rats ${ }^{47}$. In the present study, cytochrome c oxidase (CcO, complex IV) activity was diminished in the MIA-OA group. $\mathrm{CcO}$ is the terminal electron acceptor in the electron transport chain (ETC) and the regulation site for mitochondrial oxidative phosphorylation sequel ${ }^{48}$. Thus, our findings indicated that oxidative phosphorylation and mitochondrial energy homeostasis were disrupted following MIA insult. On the contrary, $\mathrm{CcO}$ was replenished in the tempol-treated rats. Likewise, a previous study has demonstrated that tempol maintained the mitochondrial respiratory function in cisplatin-induced nephrotoxicity in mice ${ }^{21}$.

In the current investigation, MIA insult accompanied the perturbed oxidative phosphorylation by oxidative stress status of lower SOD activity and elevated NOX4 contents, these results are consistent with previous studies ${ }^{11,16}$. Basically, intracellular ROS such as superoxide anion $\left(\mathrm{O}_{2}{ }^{\circ} \nabla\right)$ and $\mathrm{H}_{2} \mathrm{O}_{2}$ are derived from mitochondria and membrane NADPH oxidase (NOX) systems ${ }^{49}$. The $\mathrm{O}_{2}{ }^{*} \otimes$ reacts with biomolecules to cause molecular damage or turn spontaneously into $\mathrm{H}_{2} \mathrm{O}_{2}$ or diminished by the SOD antioxidant activity ${ }^{50}$. Inappropriately, SODs are expressed at lower levels in OA cartilage ${ }^{51}$. However, it has been reported that NOX4 is the predominant isoform active in chondrocytes of OA cartilage ${ }^{52}$. NOX4 constitutively produces $\mathrm{H}_{2} \mathrm{O}_{2}{ }^{53}$. NOX4-derived ROS generation triggers many transcriptional events responsible for inflammatory cytokines production, lipid peroxidation, mitochondrial DNA damage, MMPs production, and cartilage breakdown exacerbation ${ }^{16,54}$. Eventually, these events lead to chondrocyte cell death, osteophyte formation, synovial fibrosis, and joint pain in animal models ${ }^{55,56}$. 
Inversely, tempol post-treatment mitigated MIA-induced oxidative stress via replenishing SOD and diminishing NOX4 activity. These findings reasonably rely on the anti-oxidant efficacy of tempol. Interestingly, these results can collectively support the linkage between the anti-oxidant effect of tempol and its ability to maintain mitochondrial function dynamics and cellular redox balance which primarily emphasize the potential role of tempol in alleviating cartilage degeneration.

In this study, MIA prompted an inflammatory response that was noticeably presented by joint swelling especially during the first 10 days of the experimental period, and then subsided gradually, accompanied by edema and inflammatory cells infiltration of the synovial membrane in the related histological photomicrographs. Likewise, the MIA-induced biochemical alterations showed significant elevations of the pro-inflammatory mediators including serum IL-1 $\beta$, tissue contents of IL- 6 and NF- $\kappa B_{p 65}$, and CCL2 expressions in $\mathrm{OA}$ rats, these outcomes were previously mentioned ${ }^{6,20,57}$.

It has been noted that the abnormally generated ROS exacerbates the inflammatory process in OA and aggravates ECM and joint degradation ${ }^{10}$. Inflammation is the most significant cause of OA-related structural changes ${ }^{19}$. During the progress of $O A$, chondrocytes and synoviocytes stimulate the production of inflammatory mediators ${ }^{9}$. IL-1 $\beta$ is the most substantial inflammatory cytokine correlated to the pathogenesis of $O A^{58}$. IL-1 $\beta$ stimulates the production of pro-inflammatory IL- $6{ }^{43}$. Besides, CCL2 or called monocyte chemoattractant protein-1 (MCP-1), is well known to facilitate the migration and infiltration of monocytes and macrophages ${ }^{59}$.

In $\mathrm{OA}$, the abnormal activation of NF-KB coordinates a complex-multilayered signaling network leading to the upregulation of catabolic factors include cytokines, chemokines, prostaglandin E2, and MMPs, which eventually lead to cartilage damage and osteophytes formation ${ }^{60}$. NF-KB signal cascades cause local tissue damage, recruitment, and activation of immune cells (macrophages, lymphocytes, granulocytes), and also triggering angiogenesis ${ }^{61}$.

In the present investigation, the post-treatment of tempol subsided all the forgoing MIA-induced inflammatory mediators' release. Additionally, the treated rats exhibited marked joint swelling relief starting from the $4^{\text {th }}$ day of treatment, and histologically showed mild inflammatory cell infiltrates in the synovial membrane. Previous studies have demonstrated the anti-inflammatory effect of tempol, for example, tempol enhanced the periodontitis in a rodent model ${ }^{62}$. Also, tempol delayed the altered investigations of the collagen-induced arthritis rat model ${ }^{15}$. Accordingly, our findings indicate the potential anti-inflammatory effect of tempol on MIA-induced knee OA.

NF-KB is a key promoter in the inflammatory angiogenic pathway ${ }^{63}$. The inflammatory cells produce proangiogenic factors, such as vascular endothelial growth factor (VEGF), and fibroblast growth factor 2 (FGF 2), and thus promote the formation and invasion of new blood vessels, to facilitate inflammatory cell infiltration ${ }^{61}$. Dickkopf-related protein-1 (DKK-1), a secreted protein, is a powerful antagonist of canonical Wnt/ $\beta$-catenin signaling that is implicated in a variety of developmental and physiological 
processes ${ }^{64}$. It has been documented that increased expressions of DKK1 in temporomandibular knee $\mathrm{OA}$ in a human were positively related to VEGF and linked to the angiogenic response and cartilage matrix proteinase secretion ${ }^{65}$. Besides, upregulated DKK-1 expressions are documented to be associated with increased inflammatory cytokines, chondrocyte apoptosis, cartilage destruction, and subchondral bone deterioration in the knee of osteoarthritic rats ${ }^{66,67}$. Consistently, our results revealed elevated mRNA expression of DKK-1 in osteoarthritic rats accompanied by subchondral extravasation of blood in the related histological photomicrographs of MIA-OA group, however, DKK-1 was downregulated following tempol post-treatment signifying the potential ability of tempol in alleviating the inflammatory-dependent neovascularization.

MIA exhibits biphasic pain behavior; the early phase is the inflammatory phase (2- 6 days post-injection) and the pain is the late phase (>14 days post-injection) ${ }^{36}$. The production of chemokines, cytokines, and proteases sensitizes primary sensory neuron (PSN) afferents ${ }^{38}$. CCL2 stimulates direct excitation on nociceptive neurons and microglial activation which further triggering persistent hyperalgesia ${ }^{59}$. NF$\mathrm{KB} / \mathrm{IL}-6$ signaling activation has been documented to be interconnected with chronic pain sensitivities and cartilage damage in $\mathrm{OA}{ }^{68}$. Additionally, ROS also participates in the sensitization of the dorsal horn neurons, provoking the central sensitization through glial activation ${ }^{69}$.

Reliably, in this knee OA model, the accelerating rotarod performance test was used for the functional assessment of MIA-induced pain and motor imbalance. The osteoarthritic rats displayed poor performance in the rotarod which was presented by gradual reduction of the latency time to fall throughout the experimental schedule, these findings are in harmony with previous studies $20,26,28$.

In the current study, tempol treatment was started 7 days post-MIA injection to be enabled to assess the inflammatory-neuropathic pain interval. Besides, the histological examination shows chondrocyte degeneration/necrosis started at days 1-7 post-MIA, with increased osteoclasts and osteoblasts in subchondral bone by day $7^{70}$. Tempol-treated rats exhibited improved motor performance noticed by the significant increase of the latency time to fall on day 21 and day 28. Earlier studies have demonstrated the analgesic effects of tempol in attenuating neuropathic pain of spinal nerve ligation ${ }^{71}$ or preventing the pain sensitization after capsaicin injection into the foot ${ }^{72}$. Thus, our attained findings recommend the potential analgesic activity of tempol inferred to its anti-inflammatory effects in the MIA-induced OA model in rats. Collectively, these outcomes suggest the interconnection between the anti-oxidant effects of tempol and its ability to subside the catabolic inflammatory cascades including inflammatory cell infiltration, angiogenesis, and pain which further underline the anti-osteoarthritic role of tempol in the MIA-OA model.

OA pathogenesis is implicated by the production of abnormal levels of cytokines, chemokines, ROS, and MMPs by chondrocytes or synoviocytes which in consequence triggers direct degradation of ECM components and dysregulates chondrocyte metabolism leading to an imbalance between cartilage matrix degradation and synthesis ${ }^{61}$. Hence, chondrocytes undergo metabolic surplus of dual 
upregulations of catabolic factors secretions and anabolic factors as a compensatory repair attempt through activation of downstream signal transduction pathways ${ }^{54,73}$. Consequently, the vicious stress circles of oxidative stress and inflammation are continued in which the catabolic cascades outpace the reparative abilities of chondrocytes ultimately lead to cartilage damage ${ }^{74}$.

At the early stage of $O A$, chondrocytes stimulate TGF- $\beta$ to activate the canonical signal transducer SMAD3 directly as a repair attempt in response to cartilage degeneration ${ }^{75}$, which subsequently activates the target transcription of NOX $4^{76}$. Henceforward, the expression of NOX4 derives excessive ROS generation (especially $\mathrm{H}_{2} \mathrm{O}_{2}$ ), which activates the downstream p38MAPK phosphorylation thus resulting in deleterious signaling events ${ }^{77,78}$. Moreover, it has been documented that TGF- $\beta$ activates the non-canonical p38MAPK and NF-KB signaling by the upstream activator TGF- $\beta$-activated kinase-1 (TAK1) 79 .

The stress kinase, p38MAPK is activated in response to the inflammatory cytokines, oxidative stress, and growth factors activation ${ }^{77}$. Its activation is mediated by PKC signaling ${ }^{80}$. p38MAPK is a member of the family of serine/threonine kinases that consists of p38 kinase, c-Jun N-terminal kinase (JNK), and the extracellular signal-regulated kinases (ERKs) ${ }^{81}$. Subsequently, activation of p38MAPK triggers NF-KB signaling activity and its sequels ${ }^{82}$. Besides, it has been noted that oxidative stress-induced the phosphorylation of p38MAPK which is correlated to the activation of caspase 9 mediated apoptotic pathways ${ }^{77}$.

Hereby, our findings demonstrate that MIA injection resulted in elevations of anabolic growth factor TGF$\beta 1, \mathrm{PKC}, p$-SMAD3, and $p$-p38MAPK, and in contrast, tempol suppressed all these elevations. Relying on these findings, the anti-oxidant mechanism of tempol is not related only to its intracellular redox cycling negotiation, but it is also related to the ability of tempol to suppress the TGF- $\beta 1 /$ SMAD3/NOX4 pathway, which further diminishing NOX4-derived ROS generation sequels via suppression of $p$-p38MAPK/NF$\mathrm{KB}_{\mathrm{p} 65}$ damaging signaling cascades. These outcomes support the advance probable anti-osteoarthritic effect of tempol in promoting cartilage regeneration and opposing catabolic cascades interlinked with its anti-oxidant efficacy.

Furthermore, chondrocytes potentiate TGF- $\beta$ to secrete more IGF- 1 to boost the production of ECM synthesis proteins as an additional repair attempt to OA insult ${ }^{74}$. Thereafter, IGF-1/ IGF-1R binding activates binding sites for PI3K, which is responsible for Akt activation ${ }^{83,84}$. The continued Akt signaling is noted to deteriorate the integrity of articular cartilage, even though many reports favor the chondroprotective role of Akt ${ }^{85,86}$. The downstream Akt is phosphorylated following PI3K activation, thus affecting MMPs production via its multiple downstream target proteins such as NF-KB, GSK-3ß, mTOR, and $\mathrm{p} 53^{76}$. $\mathrm{PI} 3 \mathrm{~K} / \mathrm{Akt}$ signaling activates $\mathrm{Bax}, \mathrm{Bcl}-2$, and therefore caspase-3 expression persuading chondrocyte cell apoptosis ${ }^{87}$. Besides, PI3K/Akt activation together with PKC mediates NOX4 assembly and ROS generation ${ }^{50,88}$. PI3K/Akt/NF-KB signaling pathway has been powerfully implicated in OA inflammation ${ }^{89}$. In circumstance, $\mathrm{PI} 3 \mathrm{~K} / \mathrm{Akt}$ activates the mammalian target of rapamycin (mTOR) 
downstream target protein, which is known as a classical autophagy suppressor ${ }^{90}$. mTOR is regulated by both PI3K/Akt and MAPK signaling pathways ${ }^{87}$.

In this study, the autophagy-associated protein, beclin-1 contents were downregulated in MIA-induced OA rats. It has been suggested that beclin-1 expression was significantly downregulated in OA cartilage tissue through PI3K/Akt/mTOR signaling ${ }^{91}$. While, tempol post-treatment suppressed MIA-induced exaggerations of serum IGF-1, $p$-PI3K, and $p$-Akt, whereas, beclin-1 contents.

In summary, the potential anti-oxidant and anti-inflammatory mechanisms of tempol on MIA-induced osteoarthritis in rats can be collectively demonstrated through its ability to provoke collateral suppression

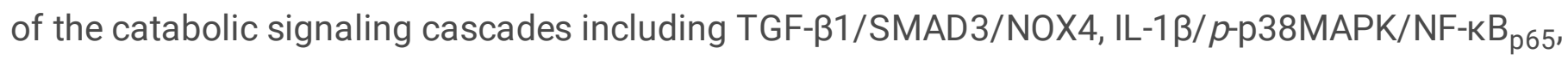
NOX4/ROS/p38MAPK/NF-kB ${ }_{\mathrm{p} 65}$, and PI3K/Akt/NF-KB $\mathrm{p}_{\mathrm{p} 5}$, which in turn results in decreasing MMPs production, reducing catabolic inflammatory sequels, limiting structural alterations, reducing angiogenesis and pain, and protecting the chondrocytes from apoptosis, besides, encouragement of chondrocytes' survival via autophagy.

Three limitations of this study are identified. First, chemical chondrotoxic agents such as MIA are reported to have unique pathophysiology that has no association with post-traumatic OA. Therefore, this method may be useful for determining whether osteoarthritic changes originate from cartilage or subchondral bone alterations and it is mainly used to observe and evaluate pain behavior and to assess the efficacy of therapeutic interventions to reduce inflammation and pain. Second, this study did not compare the tested tempol with appropriate pharmacological control. Third, this study needs a further demonstration of the autophagy picture by evaluating the lysosomal formation and/or fusion with the autophagosomes.

\section{Abbreviations}

Cartilage oligomeric matrix protein (COMP), chemotactic cytokine ligand 2 (CCL2), C-telopeptide of type II collagen (CTX-II), cytochrome c oxidase (CcO), dickkopf-related protein-1 (DKK-1), discoidin domain receptor 2 (DDR2), insulin-like growth factor-1 (IGF-1), matrix metalloproteinase-13 (MMP-13), mitogenactivated protein kinase (MAPK), monosodium iodoacetate (MIA), NADPH oxidase 4 (NOX4), nuclear factor kappa-B (NF-KB), phosphoinositide 3-kinase (PI3K), protein kinase B (Akt), protein kinase C (PKC), small mother against decapentaplegic 3 homologs (SMAD3), superoxide dismutase (SOD), and transforming growth factor- $\beta 1$ (TGF- $\beta 1$ ).

\section{Declarations}

\section{Acknowledgments}

The authors are thankful to Dr. Laila A. Rashed, Professor of Medical Biochemistry Department, Faculty of Medicine, Cairo University, Egypt, for her appreciated efforts in quantitative RT-PCR and Western blot 
procedures. The authors are also grateful to Dr. Mohamed A. Salah El-dein, Assistant professor of Histology Department, Faculty of Veterinary Medicine, Cairo University, Egypt, for his valuable assistance in histological examinations. The authors are so appreciative of Dr. Hassan Bakr and Dr. Midaa Ibrahim, veterinarians at CatDog Veterinary Hospital, Giza, Egypt, for their efforts in the radiological investigations.

\section{Author contribution}

Mahmoud M. Khattab and Rehab F. Abdel-Rahman designed the work protocol. Hagar B. Abo-zalam performed the experiments as well as data interpretation. Hagar B. Abo-zalam wrote the manuscript and prepared the figures. Rehab F. Abdel-Rahman, Mohamed F. Abd-Ellah, Rania M. Abdalsalam, Mahmoud M. Khattab revised and edited the manuscript. All authors read and approved the final manuscript.

\section{Conflict of Interests}

The authors declare that there is no conflict of interest regarding the publication of this paper.

\section{Funding sources}

This research did not receive any specific grant from funding agencies in the public, commercial, or notfor-profit sectors.

\section{Ethical Approval}

All procedures for handling, use, and euthanasia of animals were reviewed and approved by the Ethical Committee for Animal Experimentation of the Faculty of Pharmacy, Cairo University, with permit number

PT 2389, and were performed following the guides for the Care and Use of Laboratory Animals published by the US National Institutes of Health ( $8^{\text {th }}$ edition, NIH Publication, 2011).

\section{Availability of data and materials}

Datasets collected or analyzed during the current study are available from the corresponding author on request.

\section{References}

1. Goldring, M. B. \& Goldring, S. R. Osteoarthritis. J. Cell. Physiol. 213, 626-634 (2007).

2. Holyoak, D. T., Tian, Y. F., van der Meulen, M. C. H. \& Singh, A. Osteoarthritis: Pathology, Mouse Models, and Nanoparticle Injectable Systems for Targeted Treatment. Ann. Biomed. Eng. 44, 20622075 (2016).

3. Xia, B. et al. Osteoarthritis Pathogenesis: A Review of Molecular Mechanisms. Calcif. Tissue Int. 95, 495-505 (2014).

4. Takahashi, I., Matsuzaki, T. \& Hoso, M. Long-term histopathological developments in knee-joint components in a rat model of osteoarthritis induced by monosodium iodoacetate. J. Phys. Ther. Sci. 
29, 590-597 (2017).

5. Belkhodja, H. et al. Radiographic and Histopathologic Analysis on Osteoarthritis Rat Model Treated with Essential Oils of Rosmarinus officinalis and Populus alba. Pharm. Sci. 23, 12-17 (2017).

6. Lee, D. G. et al. Fucoidan Prevents the Progression of Osteoarthritis in Rats. J. Med. Food 18, 10321041 (2015).

7. Pei, Y. et al. Antioxidative nanofullerol inhibits macrophage activation and development of osteoarthritis in rats. Int. J. Nanomedicine 14, 4145-4155 (2019).

8. Wang, Y., Fan, X., Xing, L. \& Tian, F. Wnt signaling: A promising target for osteoarthritis therapy. Cell Commun. Signal. 17, 97 (2019).

9. Franz, A. et al. The role of oxidative and nitrosative stress in the pathology of osteoarthritis: Novel candidate biomarkers for quantification of degenerative changes in the knee joint. Orthop. Rev. (Pavia). 10, 7460 (2018).

10. Gundogdu, G. et al. Investigation of the efficacy of daidzein in experimental knee osteoarthritisinduced with monosodium iodoacetate in rats. Clin. Rheumatol. 39, 2399-2408 (2020).

11. Lu, J., Zhang, T., Sun, H., Wang, S. \& Liu, M. Protective effects of dioscin against cartilage destruction in a monosodium iodoacetate (MIA)-indcued osteoarthritis rat model. Biomed. Pharmacother. 108, 1029-1038 (2018).

12. Jeong, J. H. et al. Eupatilin exerts antinociceptive and chondroprotective properties in a rat model of osteoarthritis by downregulating oxidative damage and catabolic activity in chondrocytes. PLOS One 10, e0130882 (2015).

13. Kolasinski, S. L. et al. 2019 American College of Rheumatology/Arthritis Foundation Guideline for the Management of Osteoarthritis of the Hand, Hip, and Knee. Arthritis Care Res. 72, 149-162 (2020).

14. Wilcox, C. S. \& Pearlman, A. Chemistry and antihypertensive effects of tempol and other nitroxides. Pharmacol. Rev. 60, 418-469 (2008).

15. Cuzzocrea, S. et al. Beneficial effects of tempol, a membrane-permeable radical scavenger, in a rodent model of collagen-induced arthritis. Arthritis Rheum. 43, 320-328 (2000).

16. Pathak, N. N. et al. Effect of atorvastatin, a HMG-CoA reductase inhibitor in monosodium iodoacetate-induced osteoarthritic pain: Implication for osteoarthritis therapy. Pharmacol. Reports 67, 513-519 (2015).

17. Kim, J. et al. Effect of GCSB-5, a Herbal Formulation, on Monosodium lodoacetate-Induced Osteoarthritis in Rats. Evidence-Based Complement. Altern. Med. 2012, 730907 (2012).

18. Jeong, J. W. et al. Mori Folium water extract alleviates articular cartilage damages and inflammatory responses in monosodium iodoacetate-induced osteoarthritis rats. Mol. Med. Rep. 16, 3841-3848 (2017).

19. Lee, Y. M., Son, E., Kim, S. H., Kim, O. S. \& Kim, D. S. Anti-inflammatory and anti-osteoarthritis effect of Mollugo pentaphylla extract. Pharm. Biol. 57, 74-81 (2019). 
20. Yassin, N. Z. et al. Effect of a topical copper indomethacin gel on inflammatory parameters in a rat model of osteoarthritis. Drug Des. Devel. Ther. 9, 1491-1498 (2015).

21. Ahmed, L. A., Shehata, N. I., Abdelkader, N. F. \& Khattab, M. M. Tempol, a superoxide dismutase mimetic agent, ameliorates cisplatin-induced nephrotoxicity through alleviation of mitochondrial dysfunction in Mice. PLoS One 9, e108889 (2014).

22. Tanito, M., Li, F., Elliott, M. H., Dittmar, M. \& Anderson, R. E. Protective effect of TEMPOL derivatives against light-induced retinal damage in rats. Investig. Ophthalmol. Vis. Sci. 48, 1900-1905 (2007).

23. Karanovic, D. et al. Effects of single and combined losartan and tempol treatments on oxidative stress, kidney structure and function in spontaneously hypertensive rats with early course of proteinuric nephropathy. PLoS One 11, e0161706 (2016).

24. Ranjbar, A. et al. Tempol effects on diabetic nephropathy in male rats. J. Ren. Inj. Prev. 5, 74-78 (2016).

25. Ali, S. M. et al. L-Carnitine ameliorates knee lesions in mono- iodoacetate induced osteoarthritis in rats. Alexandria J. Med. 53, 61-66 (2017).

26. Choudhary, D. et al. Spinacia oleracea extract attenuates disease progression and sub-chondral bone changes in monosodium iodoacetate- induced osteoarthritis in rats. BMC Complement. Altern. Med. 18, 69 (2018).

27. Tsai, H. C., Chen, T. iang, Chen, Y. pin \& Chen, R. ming. Traumatic osteoarthritis-induced persistent mechanical hyperalgesia in a rat model of anterior cruciate ligament transection plus a medial meniscectomy. J. Pain Res. 11, 41-50 (2017).

28. El-Marasy, S. A., Abdel-Rahman, R. F. \& Abd-Elsalam, R. M. Neuroprotective effect of vildagliptin against cerebral ischemia in rats. Naunyn. Schmiedebergs. Arch. Pharmacol. 391, 1133-1145 (2018).

29. Zhang, H. et al. The Therapeutic Effects of Treadmill Exercise on Osteoarthritis in Rats by Inhibiting the HDAC3 / NF-KappaB Pathway in vivo and in vitro. Front. Physiol. 10, 1060 (2019).

30. Nishikimi, M., Appaji, N. \& Yagi, K. The occurrence of superoxide anion in the reaction of reduced phenazine methosulfate and molecular oxygen. Biochem. Biophys. Res. Commun. 46, 849-854 (1972).

31. Livak, K. J. \& Schmittgen, T. D. Analysis of Relative Gene Expression Data Using Real- Time Quantitative PCR and the 2- $\triangle \triangle C$ CT method. METHODS 25, 402-408 (2001).

32. Bradford, M. M. A rapid and sensitive method for the quantitation of microgram quantities of protein utilizing the principle of protein-dye binding. Anal. Biochem. 72, 248-254 (1976).

33. Culling, C. F. A. Handbook of Histopathological and Histochemical Techniques. (2013).

34. El-Gogary, R. I., Khattab, M. A. \& Abd-Allah, H. Intra-articular multifunctional celecoxib loaded hyaluronan nanocapsules for the suppression of inflammation in an osteoarthritic rat model. Int. J. Pharm. 583, 119378 (2020). 
35. van der Sluijs, J. A. et al. The reliability of the mankin score for osteoarthritis. J. Orthop. Res. 10, 5861 (1992).

36. Lockwood, S. M., Lopes, D. M., McMahon, S. B. \& Dickenson, A. H. Characterisation of peripheral and central components of the rat monoiodoacetate model of Osteoarthritis. Osteoarthr. Cartil. 27, 712722 (2019).

37. Guingamp, C. et al. Mono-iodoacetate-induced experimental osteoarthritis: A dose-response study of loss of mobility, morphology, and biochemistry. Arthritis Rheum. 40, 1670-1679 (1997).

38. Valente, J. D. S. The Pharmacology of Pain Associated With the Monoiodoacetate Model of Osteoarthritis. Front. Pharmacol. 10, 974 (2019).

39. Rose, B. J. \& Kooyman, D. L. A Tale of Two Joints: The Role of Matrix Metalloproteases in Cartilage Biology. Dis. Markers 2016, 4895050 (2016).

40. Pulsatelli, L., Addimanda, O., Brusi, V., Pavloska, B. \& Meliconi, R. New findings in osteoarthritis pathogenesis: therapeutic implications. Ther. Adv. Chronic Dis. 4, 23-43 (2013).

41. Kuyinu, E. L., Narayanan, G., Nair, L. S. \& Laurencin, C. T. Animal models of osteoarthritis: classification, update, and measurement of outcomes. J. Orthop. Surg. Res. 11, 19 (2016).

42. Hasegawa, A. et al. Role of Fibulin 3 in Aging-Related Joint Changes and Osteoarthritis Pathogenesis in Human and Mouse Knee Cartilage. Arthritis Rheumatol. 69, 576-585 (2017).

43. Gögebakan, B., İzmirli, M., Okuyan, H. M. \& Ataç, L. Biomarkers for Early Diagnosis of Osteoarthritis. in Osteoarthritis 1-15 (SM group, 2016).

44. Tsai, S. et al. Isorhamnetin ameliorates inflammatory responses and articular cartilage damage in the rats of monosodium iodoacetate-induced osteoarthritis. Immunopharmacol. Immunotoxicol. 41, 504-512 (2019).

45. Jeong, J. et al. Schisandrae fructus ethanol extract ameliorates inflammatory responses and articular cartilage damage in monosodium iodoacetate-induced osteoarthritis in rats. Exp. Clin. Sci. 16, 265-277 (2017).

46. Tsai, P., Lee, Y., Chen, L., Lee, C. \& Wang, C. In Vitro and In Vivo Anti-Osteoarthritis Effects of 2,3,5,4?Tetrahydroxystilbene-2-O- $\beta$-D-Glucoside from Polygonum Multifloru. molecules 23, 571 (2018).

47. Zahan, O., Serban, O., Gherman, C. \& Fodor, D. The evaluation of oxidative stress in osteoarthritis. Med. Pharm. Reports 93, 12-22 (2020).

48. Kogot-levin, A. et al. Upregulation of Mitochondrial Content in Cytochrome c Oxidase Deficient Fibroblasts. PLoS One 11, e0165417 (2016).

49. Izawa, T., Hutami, I. R., Mori, H. \& Tanaka, E. Role of Smad3 and S1P Signaling in Mandibular Condylar Cartilage Homeostasis. J. Bone Res. 5, 178 (2017).

50. Cristina, S., Valer, A., Pimenta-lopes, C., Rosa, L. \& Ventura, F. Interplay between BMPs and Reactive Oxygen Species in Cell Signaling and Pathology. Biomolecules 9, 534 (2019).

51. Gavriilidis, C., Miwa, S., Zglinicki, T. Von, Taylor, R. W. \& Young, D. A. Mitochondrial Dysfunction in Osteoarthritis Is Associated With Down-Regulation of Superoxide Dismutase 2. Arthritis Rheum. 65, 
378-387 (2013).

52. Bolduc, J. A., Collins, J. A. \& Loeser, R. F. Reactive oxygen species, aging and articular cartilage homeostasis. Free Radical Biology and Medicine vol. 132 73-82 (2019).

53. Wegner, A. M. et al. Acute Changes in NADPH Oxidase 4 in Early Post - Traumatic Osteoarthritis. J. Orthop. Res. 37, 2429-2436 (2019).

54. Mobasheri, A. et al. The role of metabolism in the pathogenesis of osteoarthritis. Nat. Rev. Rheumatol. 13, 302-311 (2017).

55. Wang, W. et al. Effects of estradiol on reduction of osteoarthritis in rabbits through effect on matrix metalloproteinase proteins. Iran. J. Basic Med. Sci. 19, 310-315 (2016).

56. Aref-eshghi, E. et al. Overexpression of MMP13 in human osteoarthritic cartilage is associated with the SMAD-independent TGF- $\beta$ signalling pathway. Arthritis Res. Ther. 17, 264 (2015).

57. Jeong, J. et al. Anti-osteoarthritic effects of ChondroT in a rat model of collagenase-induced osteoarthritis. BMC Complement. Altern. Med. 18, 131 (2018).

58. Kim, J., Yoo, J. J. \& Kim, H. A. Therapeutics in Osteoarthritis Based on an Understanding of Its Molecular Pathogenesis. Int. J. Mol. Sci. 19, 674 (2018).

59. Chen, D. et al. Osteoarthritis: toward a comprehensive understanding of pathological mechanism. Bone Res. 5, 16044 (2017).

60. Olivotto, E., Otero, M., Marcu, K. B. \& Goldring, M. B. Pathophysiology of osteoarthritis: canonical NF$\mathrm{KB} / \mathrm{IKK} \beta$-dependent and kinase-independent effects of IKKa in cartilage degradation and chondrocyte differentiation. Rheum. Muscloskeletal Dis. 1, e000061 (2015).

61. Henrotin, Y., Pesesse, L. \& Lambert, C. Targeting the synovial angiogenesis as a novel treatment approach to osteoarthritis. Ther. Adv. Musculoskelet. Dis. 6, 20-34 (2014).

62. Di Paola, R. et al. Effects of Tempol, a membrane-permeable radical scavenger, in a rodent model periodontitis. J. Clin. Periodontol. 32, 1062-1068 (2005).

63. Ridiandries, A., Tan, J. T. M. \& Bursill, C. A. The role of CC-chemokines in the regulation of angiogenesis. Int. J. Mol. Sci. 17, 1856 (2016).

64. Jiang, S. jun, Li, W., Li, Y. J., Fang, W. \& Long, X. Dickkopf - related protein 1 induces angiogenesis by upregulating vascular endothelial growth factor in the synovial fibroblasts of patients with temporomandibular joint disorders. Mol. Med. Rep. 12, 4959-4966 (2015).

65. Jiang, S. jun, Jin, Z. zhi \& Peng, Y. jian. Expression of Dickkopf-related Protein 1 in Patients with Temporomandibular Osteoarthritis after Treatment with Hyaluronic Acid. Curr. Med. Sci. 40, 574-579 (2020).

66. Weng, L. H., Wang, C. J., Ko, J. Y., Sun, Y. C. \& Wang, F. S. Control of Dkk-1 ameliorates chondrocyte apoptosis, cartilage destruction, and subchondral bone deterioration in osteoarthritic knees. Arthritis Rheum. 62, 1393-1402 (2010).

67. Weng, L. et al. Inflammation induction of Dickkopf-1 mediates chondrocyte apoptosis in osteoarthritic joint. Osteoarthr. Cartil. 17, 933-943 (2009). 
68. Bowles, R. D. et al. In Vivo Luminescent Imaging of NF-kB Activity and Serum Cytokine Levels Predict Pain Sensitivities in a Rodent Model Of Osteoarthritis. Arthritis Rheumatol. 66, 637-646 (2014).

69. Lee, Y. et al. Monosodium iodoacetate-induced joint pain is associated with increased phosphorylation of mitogen activated protein kinases in the rat spinal cord. Mol. Pain 7, 39 (2011).

70. Zhang, R. X., Ren, K. \& Dubner, R. Osteoarthritis pain mechanisms: Basic studies in animal models. Osteoarthr. Cartil. 21, 1308-1315 (2013).

71. Jia, D., Wang, H., Han, B., Zhang, L. \& Guo, J. Tempol Attenuates Neuropathic Pain by Inhibiting Nitric Oxide Production. Anal. Cell. Pathol. (Amst). 2019, 8253850 (2019).

72. Schwartz, E. S., Lee, I., Chung, K. \& Chung, J. M. Oxidative stress in the spinal cord is an important contributor in capsaicin-induced mechanical secondary hyperalgesia in mice. Pain 138, 514-524 (2008).

73. Goldring, M. B. et al. Roles of inflammatory and anabolic cytokines in cartilage metabolism: signals and multiple effectors converge upon MMP-13 regulation in osteoarthritis. Eur. Cells Mater. 21, 202220 (2011).

74. Wei, F., Lee, J. K., Wei, L., Qu, F. \& Zhang, J. Correlation of insulin-like growth factor 1 and osteoarthritic cartilage degradation: a spontaneous osteoarthritis in guinea-pig. Eur. Rev. Med. Pharmacol. Sci. 21, 4493-4500 (2017).

75. Kumar, A., Dutta Choudhury, M., Ghosh, P. \& Palit, P. Discoidin domain receptor 2: An emerging pharmacological drug target for prospective therapy against osteoarthritis. Pharmacol. Reports 71, 399-408 (2019).

76. Chen, J., Crawford, R. \& Xiao, Y. Vertical inhibition of the PI3K/Akt/mTOR pathway for the treatment of osteoarthritis. J. Cell. Biochem. 114, 245-249 (2013).

77. Takebe, K. et al. Regulation of p38 MAPK phosphorylation inhibits chondrocyte apoptosis in response to heat stress or mechanical stress. Int. J. Mol. Med. 27, 329-335 (2011).

78. Yan, S. et al. Down-regulation of protease-activated receptor 2 ameliorated osteoarthritis in rats through regulation of MAPK/NF-KB signaling pathway in vivo and in vitro. Biosci. Rep. 40, BSR20192620 (2020).

79. Boehme, K. A. \& Rolauffs, B. Onset and progression of human osteoarthritis-Can growth factors, inflammatory cytokines, or differential miRNA expression concomitantly induce proliferation, ECM degradation, and inflammation in articular cartilage? International Journal of Molecular Sciences vol. 192282 (2018).

80. Loegering, D. J. \& Lennartz, M. R. Protein kinase C and toll-like receptor signaling. Enzyme Res. 2011, 537821 (2011).

81. Wen, Z. et al. Glucosamine sulfate reduces experimental osteoarthritis and nociception in rats: association with changes of mitogen-activated protein kinase in chondrocytes. Osteoarthr. Cartil. 18, 1192-1202 (2010).

82. Yuan, J., Ding, W., Wu, N., Jiang, S. \& Li, W. Protective Effect of Genistein on Condylar Cartilage through Downregulating NF-кB Expression in Experimentally Created Osteoarthritis Rats. Biomed 
Res. Int. 2019, 2629791 (2019).

83. Massicotte, F. et al. Abnormal insulin-like growth factor 1 signaling in human osteoarthritic subchondral bone osteoblasts. Arthritis Res. Ther. 8, R177 (2006).

84. Zhang, Z. et al. The effects of different doses of IGF-1 on cartilage and subchondral bone during the repair of full-thickness articular cartilage defects in rabbits. Osteoarthr. Cartil. 25, 309-320 (2017).

85. Li, H. et al. TNF-aincreases the expression of inflammatory factors in synovial fibroblasts by inhibiting the PI3K/AKT pathway in a rat model of monosodium iodoacetate-induced osteoarthritis. Exp. Ther. Med. 16, 4737-4744 (2018).

86. Xie, J. et al. Sustained Akt signaling in articular chondrocytes causes osteoarthritis via oxidative stress-induced senescence in mice. Bone Res. 7, 23 (2019).

87. Wu, Z. et al. Chondro-protective effects of polydatin in osteoarthritis through its effect on restoring dysregulated autophagy via modulating MAPK, and PI3K / Akt signaling pathways. Sci. Rep. 9, 13906 (2019).

88. Nakanishi, A., Wada, Y., Kitagishi, Y. \& Matsuda, S. Link between PI3K/AKT/PTEN pathway and NOX protein in diseases. Aging Dis. 5, 203-211 (2014).

89. Ju, L. et al. Huoxuezhitong capsule ameliorates MIA-induced osteoarthritis of rats through suppressing PI3K/ Akt/ NF-kB pathway. Biomed. Pharmacother. 129, 110471 (2020).

90. Wang, F. et al. IL-1 $\beta$ receptor antagonist ( IL-1Ra ) combined with autophagy inducer ( TAT- Beclin1 ) is an effective alternative for attenuating extracellular matrix degradation in rat and human osteoarthritis chondrocytes. Arthritis Res. Ther. 21, 171 (2019).

91. Song, B. et al. Beclin 1 overexpression inhibits chondrocyte apoptosis and downregulates extracellular matrix metabolism in osteoarthritis. Mol. Med. Rep. 16, 3958-3964 (2017).

\section{Table}

Table.1. The primer sequences for analyzing mRNA expression of CCL2, DDR2, DKK-1, fibulin3 , and PKC.

\begin{tabular}{|c|l|}
\hline mRNA species & \multicolumn{1}{|c|}{ Primer sequence $\left(5^{\prime}-3^{\prime}\right)$} \\
\hline CCL2 & $\begin{array}{l}\text { F: AGCCAACTCTCACTGAAGC } \\
\text { R: GTGAATGAGTAGCAGCAGGT }\end{array}$ \\
\hline DDR2 & $\begin{array}{l}\text { F: CTCCCAGAATTTGCTCCAG } \\
\text { R: GCCACATCTTTCCTGAGA }\end{array}$ \\
\hline \multirow{2}{*}{ DKK-1 } & $\begin{array}{l}\text { F: CAACGCTATCAAGAACCTGC } \\
\text { R: GATCTTGGACCAGAAGTGTC }\end{array}$ \\
\hline \multirow{2}{*}{ Fibulin-3 } & $\begin{array}{l}\text { F: TGTGACCCAGGATATGAACTTGAG } \\
\text { R: AGCCCCCTTGTAGATTGTAGCA }\end{array}$ \\
\hline PKC & $\begin{array}{l}\text { F: TGAATCCTCAGTGGAATGAGT } \\
\text { R: GGTTGCTTTCTGTCTTCTGAA }\end{array}$ \\
\hline \multirow{3}{*}{$\beta$-actin } & $\begin{array}{l}\text { F: CCAACCGCGAGAAGATGA } \\
\text { R: CCAGAGGCGTACAGGGATAG }\end{array}$ \\
\hline
\end{tabular}


Where, CCL2: chemotactic cytokine ligand 2, DDR2: discoidin domain receptor 2, DKK-1: dickkopf-related protein-1, F: forward primer, mRNA: messenger RNA, PKC: protein kinase C, R: reverse primer.

\section{Figures}

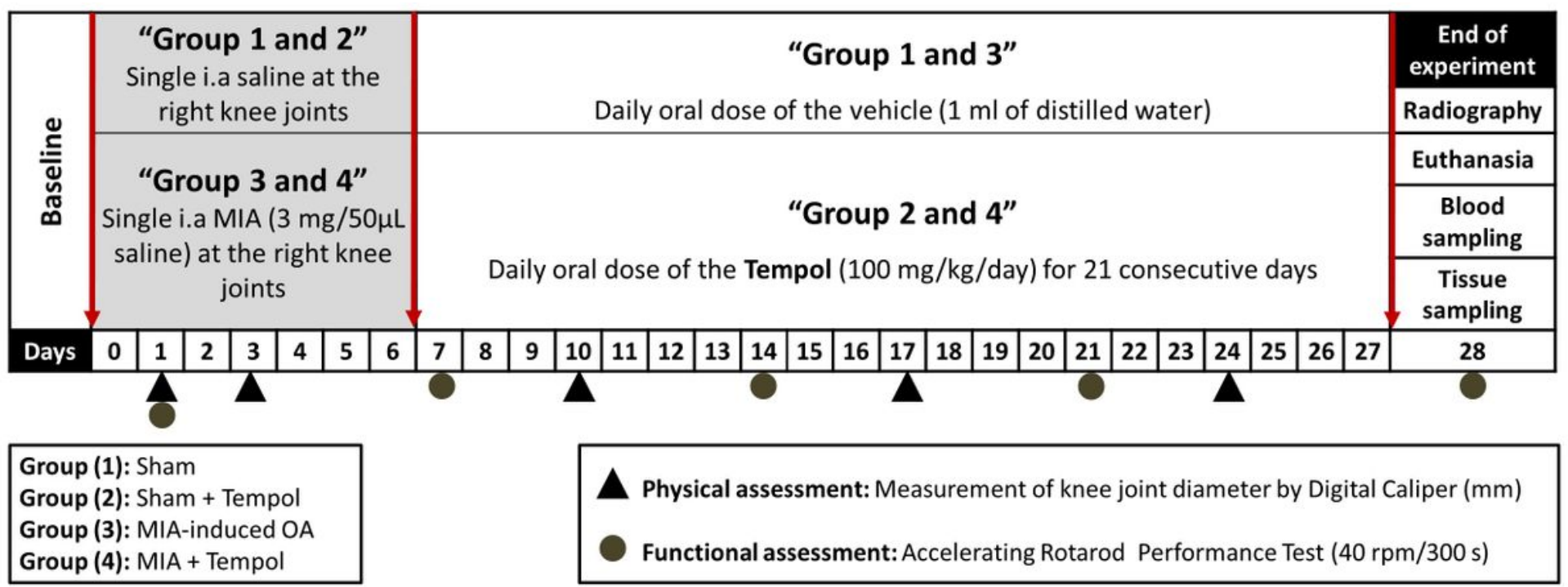

\section{Figure 1}

Schematic diagram of the experimental protocol. Rats were randomly assigned into four groups. In group (1): normal rats were subjected to a single intra-articular injection of normal saline in their right knee joints on day 0 and then subjected to a daily $1 \mathrm{ml}$ of distilled water by oral gavage, one week after saline injection for 21 days. In group 2: rats were exposed to intra-articular injection of normal saline in their right knee joints on day 0 and then administered tempol $(100 \mathrm{mg} / \mathrm{kg} /$ day) by oral gavage starting from the 7th day of the experiment for 21 consecutive days. In group 3: OA was induced by a single unilateral intra-articular injection of MIA on day 0 in a dose of $3 \mathrm{mg}$ dissolved in $50 \mu \mathrm{L}$ saline. In group 4: osteoarthritic rats were administered tempol starting from the 7th day of the experiment in a dose of (100 $\mathrm{mg} / \mathrm{kg} /$ day) by oral gavage for 21 consecutive days. Where, MIA: monosodium iodoacetate, $\mathrm{mm}$ : millimeter, OA: osteoarthritis, and rpm: rotations per minute. 
(a)

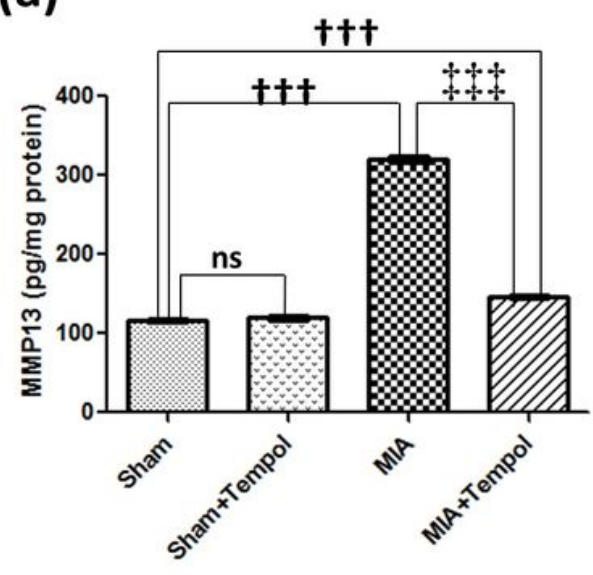

(d)

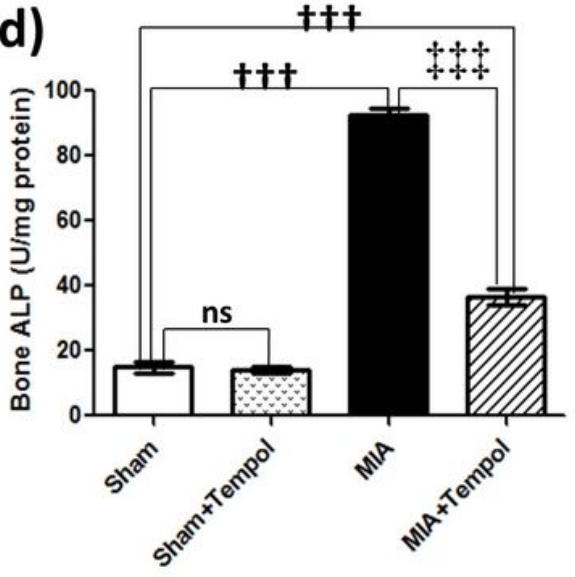

(b)

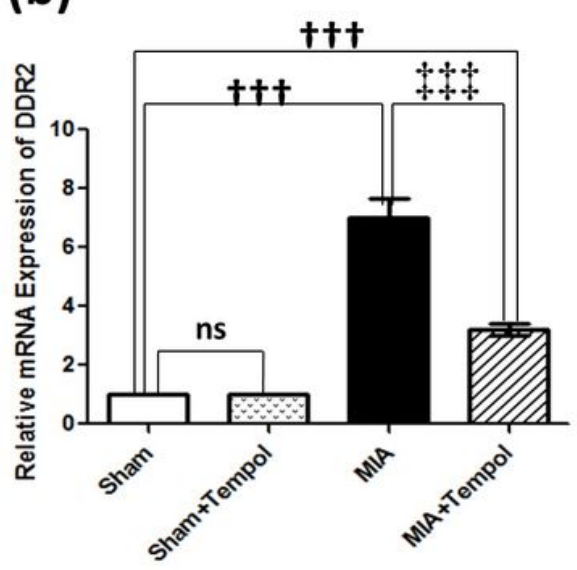

(e)

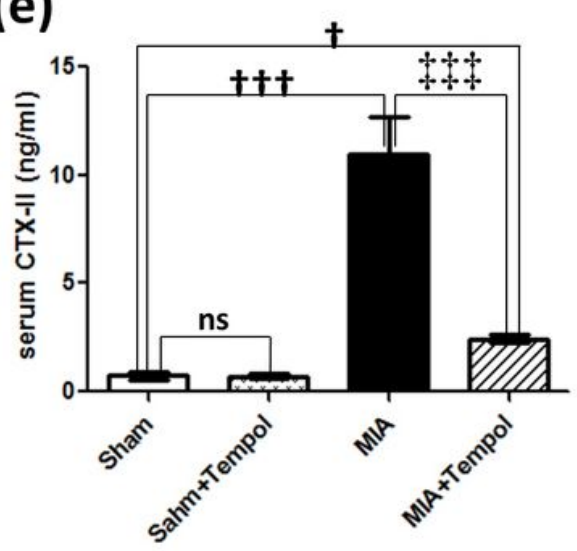

(c)

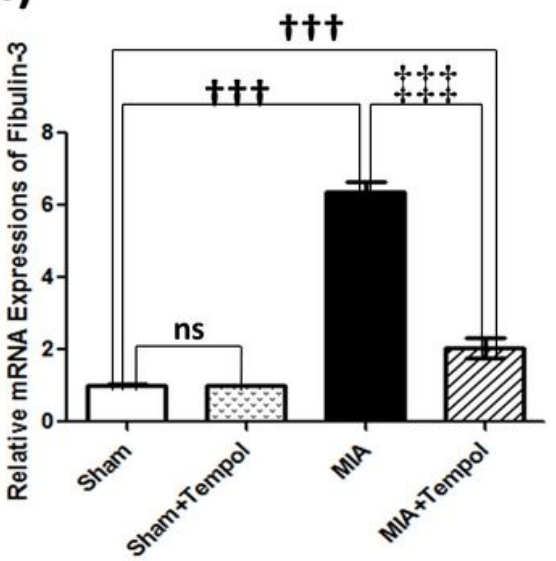

(f)

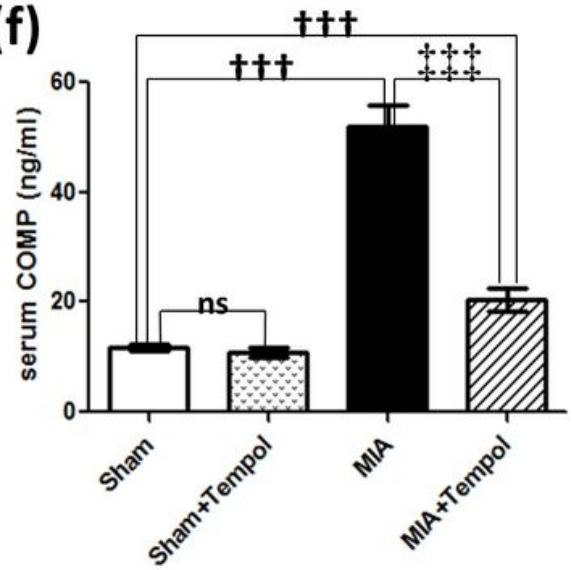

Figure 2

Effect of tempol on knee joint degradation-related biomarkers on MIA-OA rats. Rats were subjected to a single intra-articular injection of $3 \mathrm{mg} \mathrm{MIA} / 50 \mu \mathrm{L}$ saline in their right knees, and then tempol was administered starting from the 7th day of the experiment in a dose of $(100 \mathrm{mg} / \mathrm{kg} / \mathrm{day})$ by oral gavage for 21 consecutive days. (a) Tissue contents of metalloproteinase-13 (MMP-13), (b) The mRNA expression of discoidin domain receptor 2 (DDR2), (c) The mRNA expression of fibulin-3, (d) Tissue contents of bone alkaline phosphatase (ALP), (e) The serum levels of crosslinked C-telopeptides of type II collagen (CTX-II), and (f) The serum levels of cartilage oligomeric matrix protein (COMP). Statistical analysis was carried out using one-way ANOVA followed by Tukey's multiple comparison test. Results are expressed as mean $\pm S D(N=6)$. $+P<0.05$ and $+\dagger+P<0.001$ vs. the sham group, and $\neq \neq \neq P<0.001$ vs. MIAinduced OA group. 

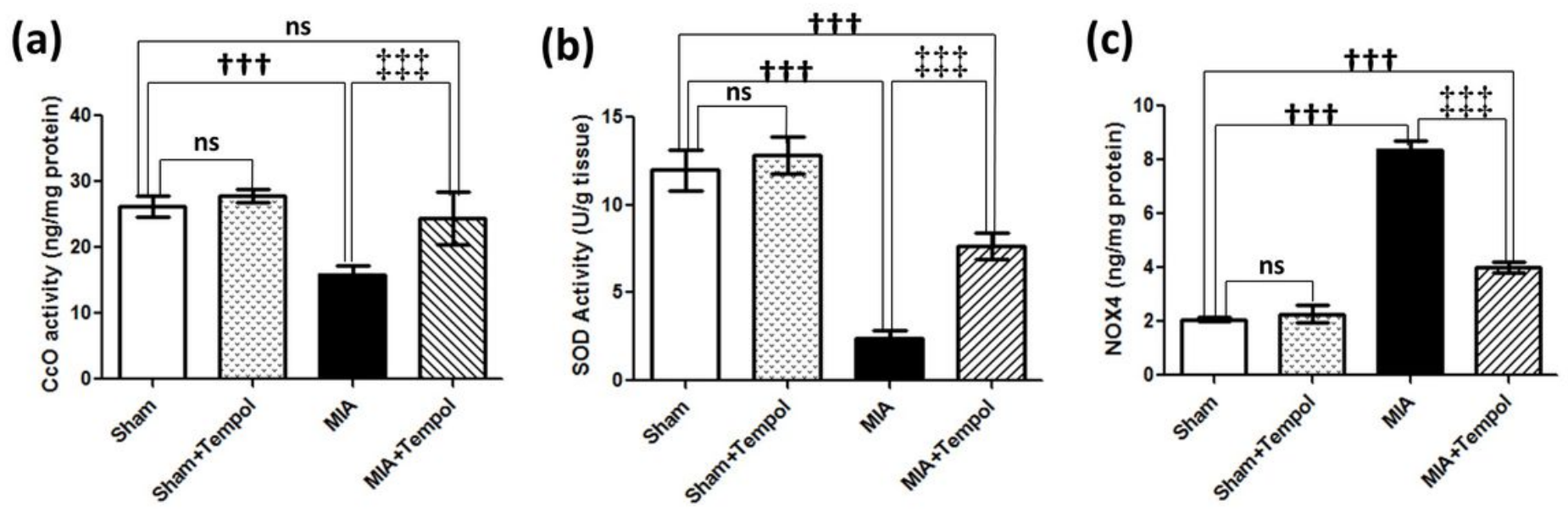

Figure 3

Effect of tempol on Complex IV oxidase and oxidative stress on MIA-OA rats. Rats were subjected to a single intra-articular injection of $3 \mathrm{mg} \mathrm{MIA} / 50 \mu \mathrm{L}$ saline in their right knees, and then tempol was administered starting from the 7th day of the experiment in a dose of $(100 \mathrm{mg} / \mathrm{kg} /$ day $)$ by oral gavage for 21 consecutive days. (a) Tissue contents of cytochrome c oxidase (CcO) or Complex IV, (b) Tissue contents of superoxide dismutase (SOD), and (c) Tissue contents of NADPH oxidase 4 (NOX4). Statistical analysis was carried out using one-way ANOVA followed by Tukey's multiple comparison test. Results are expressed as mean $\pm S D(N=6)$. $+\dagger+P<0.001$ vs. the sham group, and $\ddagger \neq ¥ P<0.001$ vs. MIA-induced $O A$ group. 
(a) "Physical Assessment"

Digital Calibration of Knee Joint Diameter

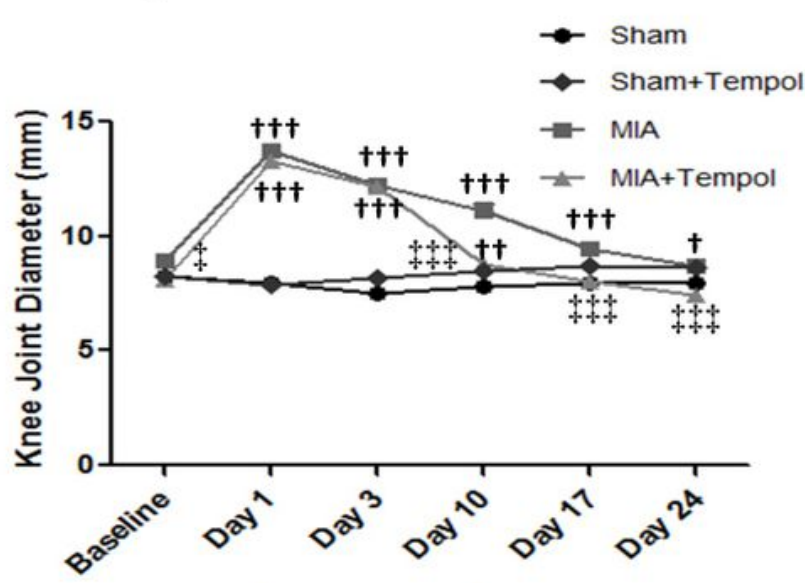

Experiment days (b) "Functional Assessment"

Accelerating Rotarod Performance Test

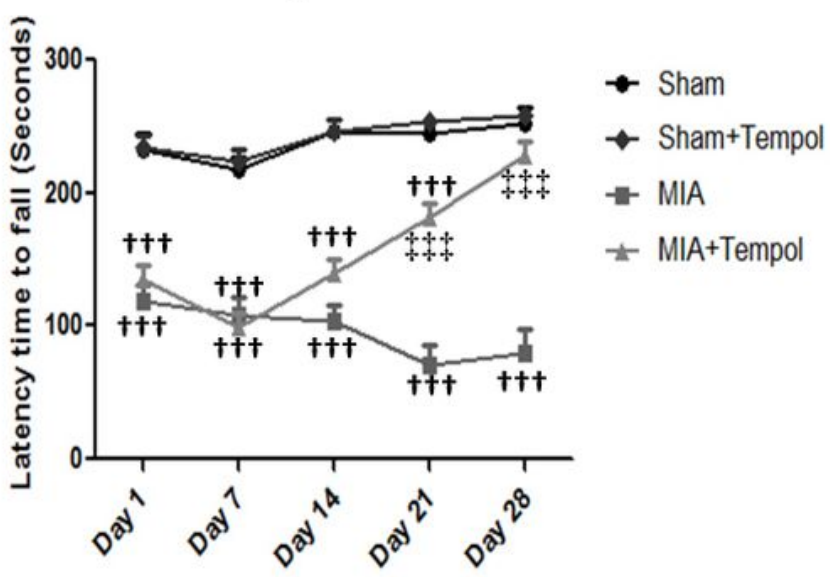

Experiment days
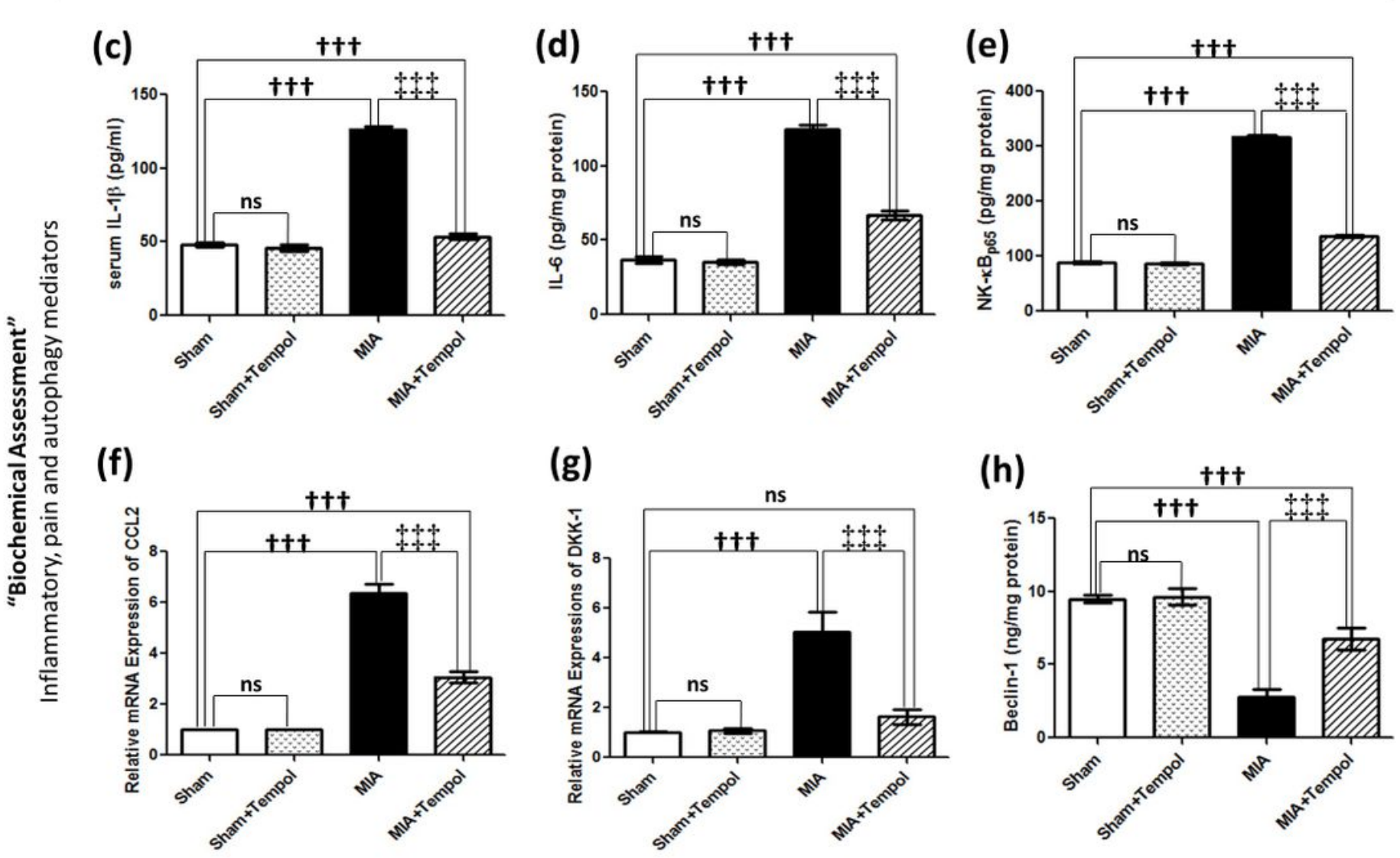

(g)

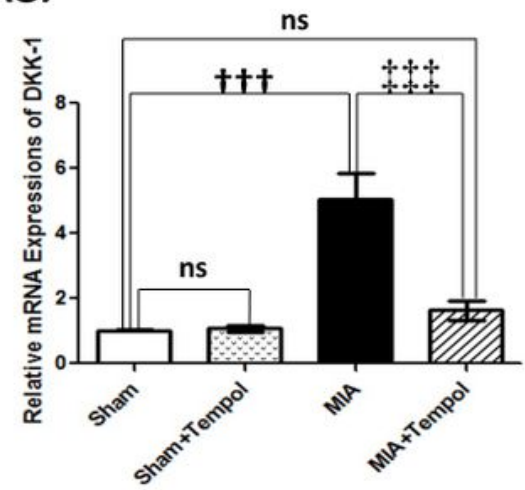

(h)

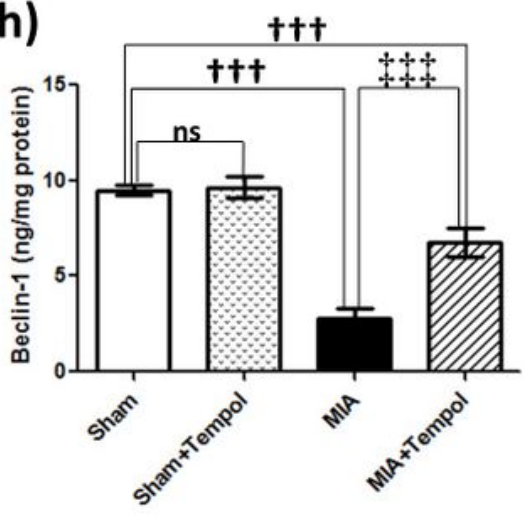

Figure 4

Effect of tempol on the catabolic inflammatory cascade on MIA-OA rats. Rats were subjected to a single intra-articular injection of $3 \mathrm{mg} \mathrm{MIA} / 50 \mu \mathrm{L}$ saline in their right knees, and then tempol was administered starting from the 7th day of the experiment in a dose of $(100 \mathrm{mg} / \mathrm{kg} / \mathrm{day})$ by oral gavage for 21 consecutive days. Values of (a) knee joint diameter and (b) latency time to fall are expressed as mean \pm SD $(\mathrm{N}=8)$. Statistical analysis was carried out using repeated measures ANOVA test for factors time and 
group, followed by the Bonferroni test. Whereas, values of (c) The serum levels of Interleukin-1 $\beta$ (IL-1 $\beta$ ), (d) Tissue contents of Interleukin-6 (IL-6), (e) Tissue contents of nuclear factor-kappa B (NF-kBp65), (f) The mRNA expression of chemotactic cytokine ligand 2 (CCL2), (g) The mRNA expression of dickkopf related protein-1 (DKK-1), and (h) beclin-1, are expressed as mean $\pm S D(N=6)$. Statistical analysis was carried out using one-way ANOVA followed by Tukey's multiple comparison test. $+P<0.05,++P<0.01$, $+†+P<0.001$ vs. the sham group, and $\ddagger \mathrm{P}<0.05$, $\neq \neq \neq \mathrm{P}<0.001$ vs. MIA-induced $\mathrm{OA}$ group.
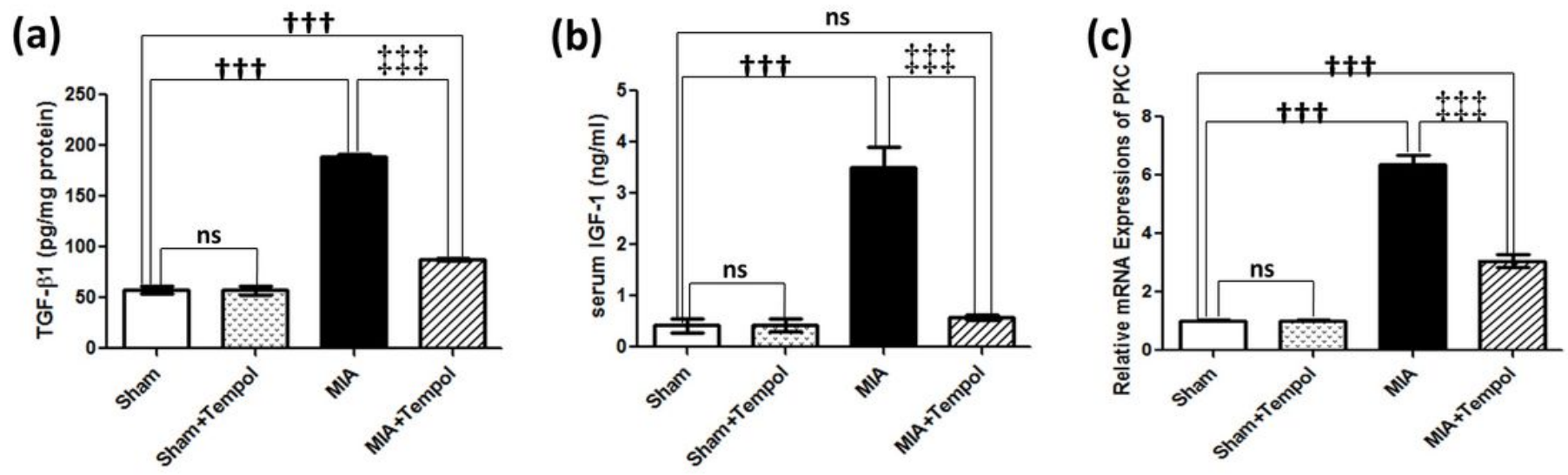

(d)

(e)
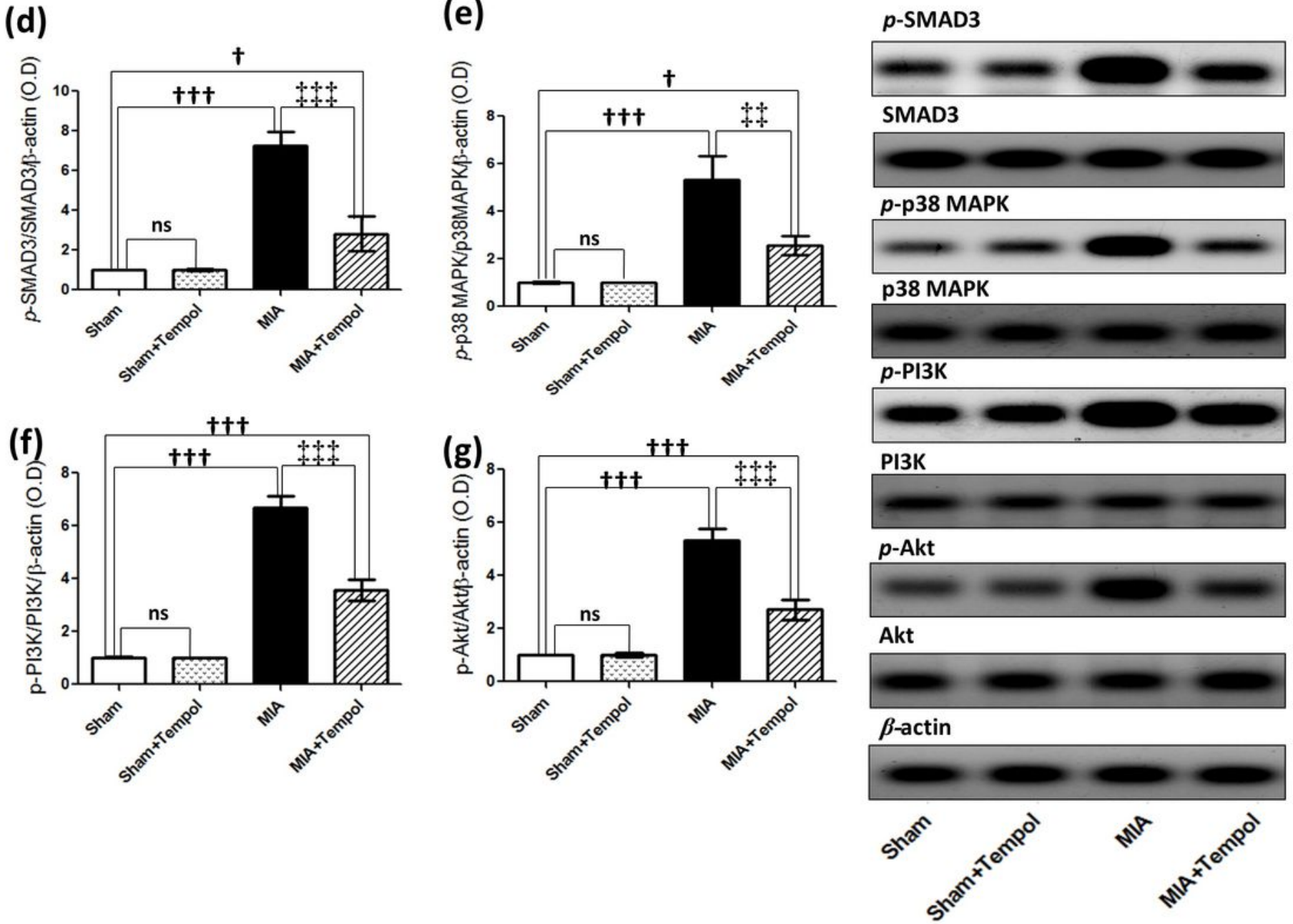

Figure 5 
Effect of tempol on growth factors and intracellular signaling kinases on MIA-OA rats. Rats were subjected to a single intra-articular injection of $3 \mathrm{mg} \mathrm{MIA} / 50 \mu \mathrm{L}$ saline in their right knees, and then tempol was administered starting from the 7th day of the experiment in a dose of ( $100 \mathrm{mg} / \mathrm{kg} /$ day) by oral gavage for 21 consecutive days. (a) Tissue contents of transforming growth factor- $\beta 1$ (TGF- $\beta 1$ ), (b) The serum levels of insulin-like growth factor-1 (IGF-1), (c) The mRNA expression of protein kinase $C$ (PKC), (d) The protein expression of the phosphorylated small mother against decapentaplegic 3 homologs (p-SMAD3), (e) The protein expression of phosphorylated p38 mitogen-activated protein kinase (p-p38MAPK), (f) The protein expression of phosphorylated phosphoinositide 3-kinase (p-PI3K), and (g) The protein expression of phosphorylated protein kinase $B$ (p-Akt). The cropped blots of p-SMAD3, $p$ p38MAPK, $\mathrm{p}-\mathrm{PI} 3 \mathrm{~K}$, and $\mathrm{p}$-Akt were presented relative to that of $\beta$-actin, and the uncropped images are available in the Supplementary File. Statistical analysis was carried out using one-way ANOVA followed by Tukey's multiple comparison test. Results are expressed as mean $\pm S D(N=6)$. $+P<0.05$ and $\dagger+† P<0.001$ vs. the sham group, and $¥ \neq P<0.01$ and $\neq \neq \neq P<0.001$ vs. MIA-induced $O A$ group. 

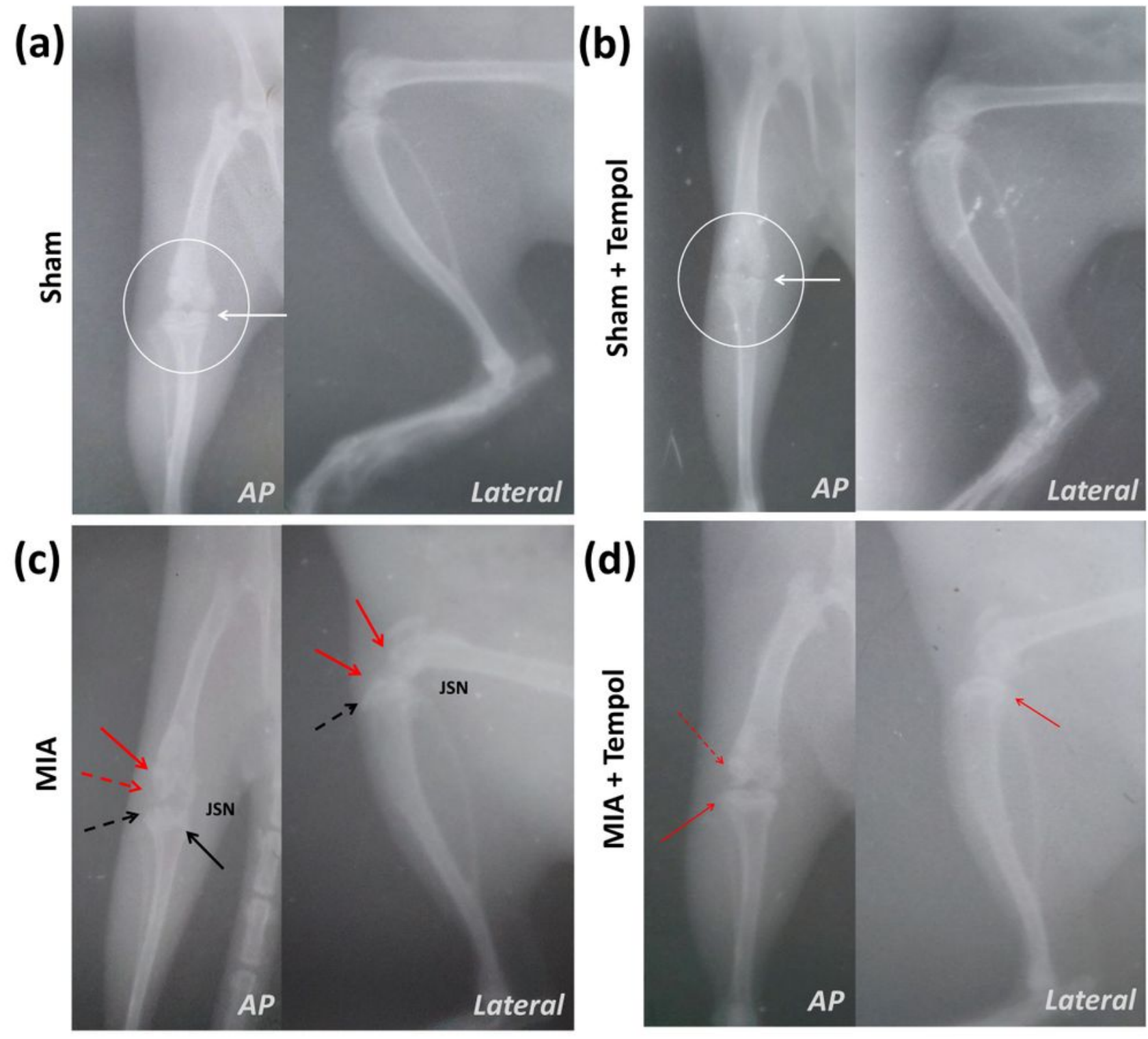

(d)

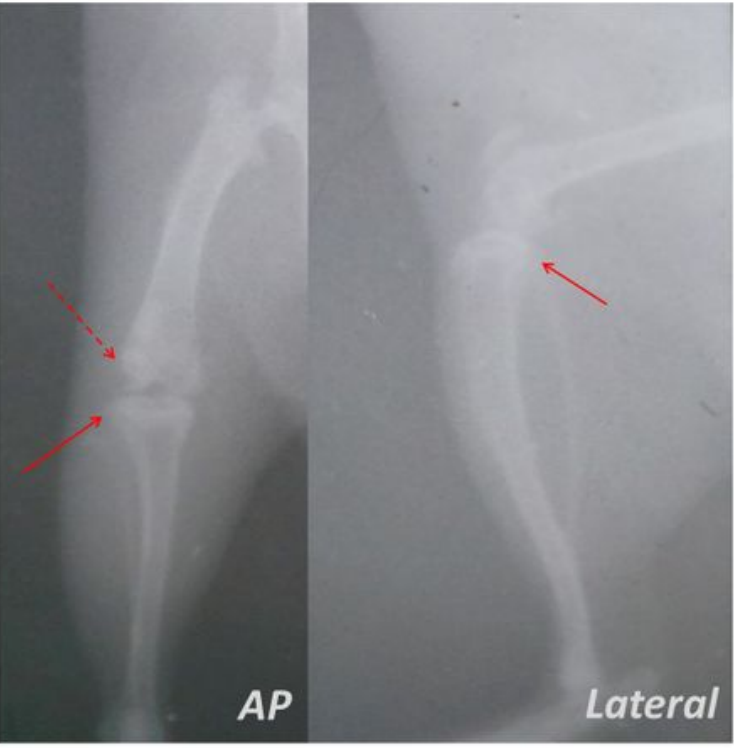

- White arrow $=$ Normal femero-tibial joint spacing

- White circle $=$ Normal surface of the femoral condyles and proximal tibia

- Red arrow = Osteophytes

- Black arrow = Joint opacity

- Red dashed arrow $=$ Roughness of the femoral condyles surface

- Black dashed arrow $=$ Roughness of the proximal tibia surface

- JSN= Joint Space Narrowing

\section{Figure 6}

Representative X-ray radiographs. Normal radiographs were revealed in the sham group (a) and Sham+Tempol group (b) along the hip-knee-ankle (HKA) axis. However, the images of the MIA-induced OA group (c) showed increased joint opacity (black arrow), and osteophyte formation (red arrow) associated with rough surfaces of the femoral condyles and proximal tibia (red and black dashed arrow). On the other hand, the X-ray images of the tempol post-treated group (d) showed almost normal joint space with 
little lysis of the femoral articular surface (red dashed arrow) and few osteophytes (red arrow). Where, AP view: anterior-posterior view (Flexion angle $10^{\circ}$ ), and lateral view (Flexion angle $30^{\circ}$ ).

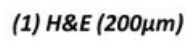
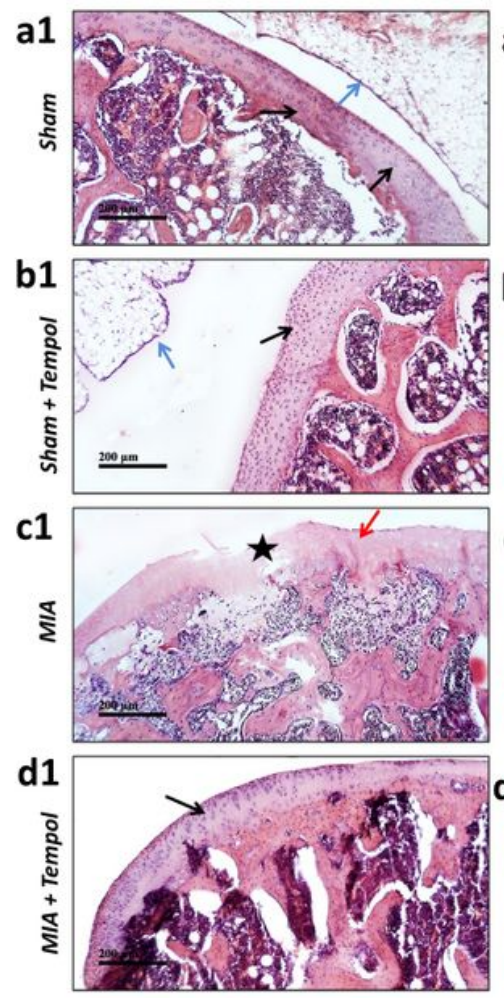

- Black arrow = Intact chondrocytes

- Blue arrow = Intact synovial membrane

- Red arrow $=$ Degenerated chondrocytes

- Black star $=$ Focal erosions and fissures in the articular cartilage

- Red star $=$ Subchondral extravasation of blood

- Red dashed arrow = Inflammatory cells infiltrates and edema (in the synovial membrane)

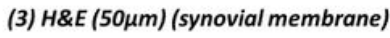
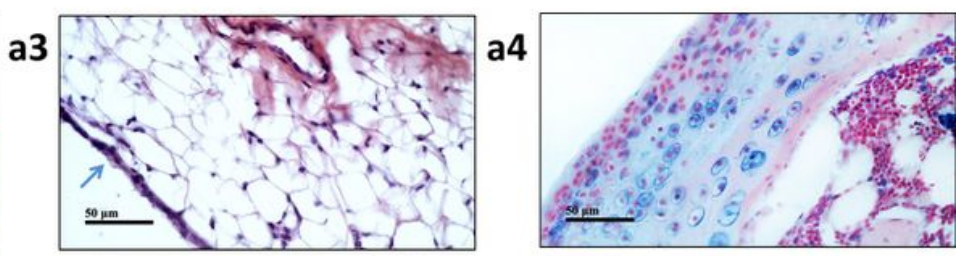

b3
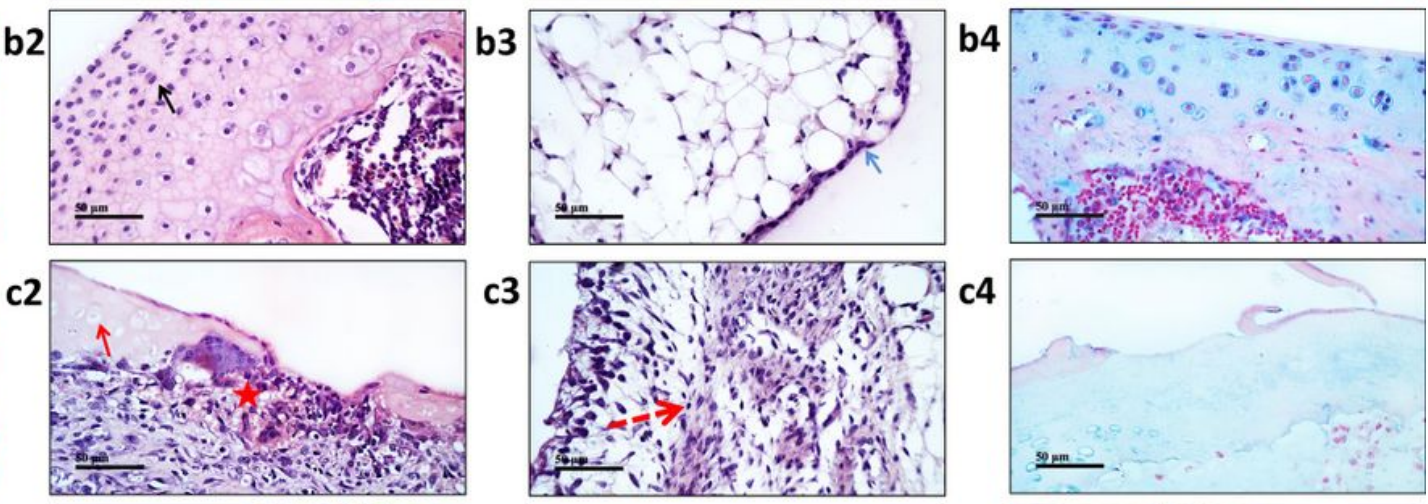

c3

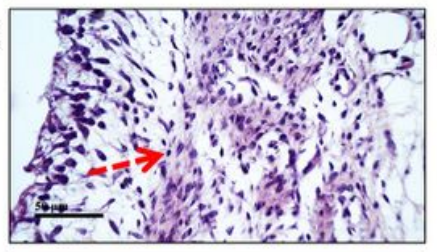

c4

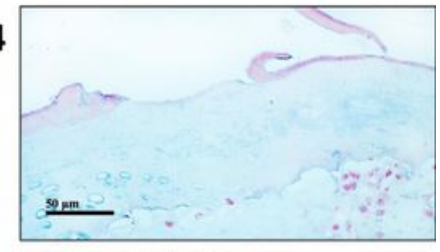

d3

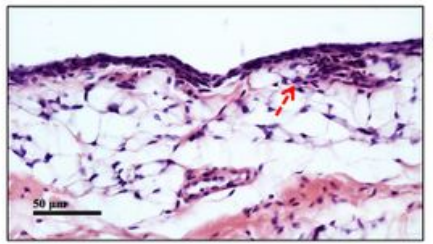

d4

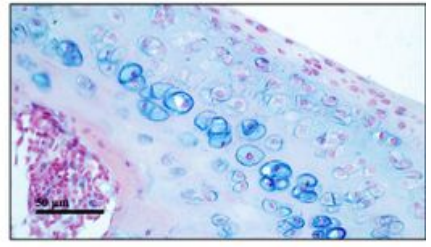

ns

(e)

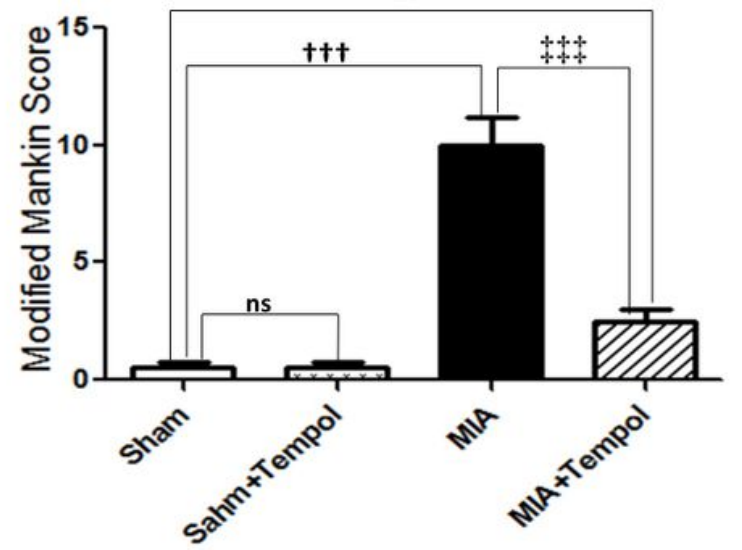

Figure 7

Representative H\&E and alcian blue histopathological photomicrographs. The micrographs of the sham groups (a\&b) revealed normal histological structures of articular cartilage layers with apparent intact isogenous chondrocytes (black arrow) and regular smooth articular surfaces. Intact synovial membranes and normal blood vessels are recorded as a blue arrow in the sections of (a3\&b3). Higher proteoglycans reactivity to alcian blue staining was also observed in (a4\&b4). The worst distortion is observed in MIAinduced OA sections, where severe degenerative and necrotic changes with significant loss of many chondrocytes (red arrow) are accompanied by many focal erosions and fissures of articular cartilage superficial zones (black star) in (c1) photomicrograph with occasional subchondral extravasation of blood (red star) in (c2). Significant edema of synovial membranes with many inflammatory cells 
infiltrates (red dashed arrow) were recorded in (c3). The MIA-induced OA demonstrated severe loss of proteoglycans reactivity to alcian blue staining all over the articular surfaces (c4). Photomicrographs of the tempol post-treated group (d1\&d2) showed more intact chondrocytes (black arrows) and regular smooth articular surfaces. Mild inflammatory cell infiltrates (red dashed arrow) were also observed in synovial membranes (d3). Post-treatment with tempol (d4) retained the higher proteoglycan reactivity. Panel (e) represents the means of the modified Mankin scoring system (Score range: 0-13, from normal cartilage to the maximal score of osteoarthritis), where the statistical analysis was carried out using oneway ANOVA followed by Tukey's multiple comparison test. Results are expressed as mean $\pm S D(N=3)$. $\dagger+† P<0.001$ vs. the sham group, and $\neq \neq \neq P<0.001$ vs. MIA-induced $O A$ group.

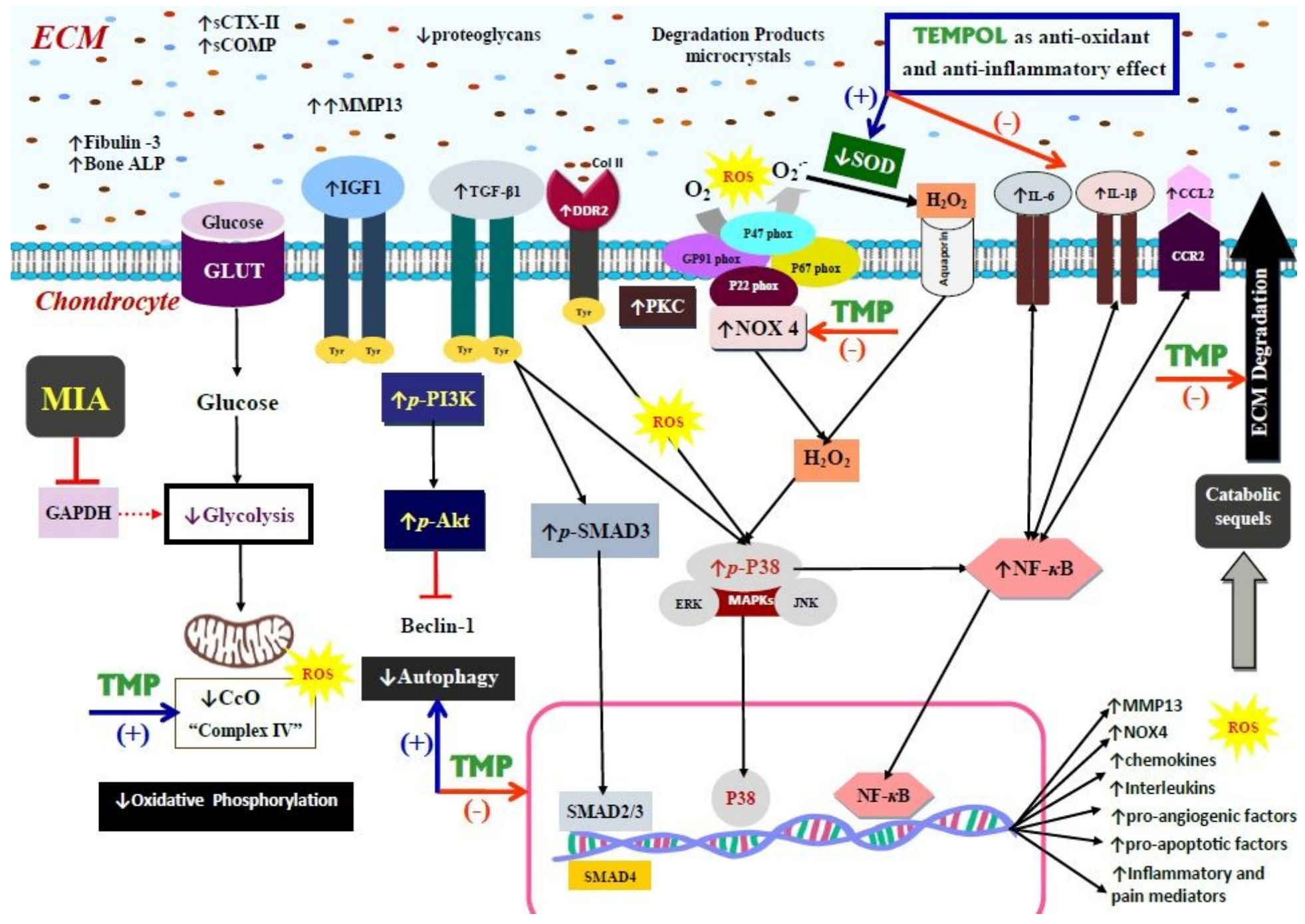

Figure 8

Schematic portrayal of the related pathological aspects of monosodium iodoacetate (MIA) and the potential therapeutic strategies of tempol (TMP) on osteoarthritic rats. MIA inhibits glyceraldehyde-3phosphate dehydrogenase (GAPDH) activity leading to disruption of glycolysis and aerobic cellular respiration. MIA-OA insult tracks chondrocyte toward a special pattern of reactive oxygen species (ROS) production, catabolic inflammatory cascades, and chondrocyte clustering involving the production of both extracellular matrix proteins and matrix-degrading enzymes. Subsequently, catabolic-degrading 
effects overwhelm the anabolic-protective function in OA chondrocytes which trails deleterious signaling pathways ultimately leading to cartilage degeneration. Tempol, a redox-cycling agent, which possesses superoxide dismutase mimetic and catalase-like actions, protects the articular cartilage from MIA destructive insult chiefly by the maintenance of mitochondrial function dynamics through replenishing mitochondrial complex IV oxidase and conservation of chondrocyte redox balance by reducing ROS production. Besides, tempol adopted interlinked mechanisms such as anti-inflammatory and analgesic effects, maintenance of chondrocyte metabolism, reducing extracellular matrix (ECM) degradation, supporting autophagy, protecting from apoptosis, and diminishing collateral intracellular downstream signaling pathways such as [TGF-31/SMAD3/NOX4], [NOX4-derived ROS/p38MAPK/NF-kBp65], [IL-1ß/pp38MAPK/NF-KBp65], and [PI3K/Akt/NF-kBp65]. Where, bone ALP: bone alkaline phosphatase, CCL2: chemotactic cytokine ligand 2, CCR2: chemokine (C-C motif) receptor 2, Col II: collagen type II, sCOMP: serum cartilage oligomeric matrix protein, $\mathrm{CcO}$ : cytochrome c oxidase (complex IV), sCTX-II: serum Ctelopeptide of type II collagen, DDR2: discoidin domain receptor 2, ECM: extracellular matrix, ERK: extracellular signal-regulated kinases, GLUT: glucose transporter, H2O2: hydrogen peroxides, IGF-1: insulin-like growth factor-1, IL-1 $\beta$ : interleukin-1 $\beta$, IL-6: interleukin-6, JNK: c-Jun N-terminal kinase, MIA: monosodium iodoacetate, MMP-13: matrix metalloproteinase-13, NF-kB: nuclear factor kappa-B, NOX4: NADPH oxidase 4, $02^{\circ}$ : : superoxide anion, OA: osteoarthritis, p-Akt: phosphorylated protein kinase $B$, PKC: protein kinase C, p-p38 MAPK: phosphorylated p38 mitogen-activated protein kinase, p-PI3K: phosphorylated phosphoinositide 3-kinase, ROS: reactive oxygen species, SMAD3: small mother against deapentaplegic 3 superfamily, SOD: superoxide dismutase enzyme, TGF- $\beta 1$ : transforming growth factor$\beta 1$, and TMP: tempol, Tyr: tyrosine kinase receptor.

\section{Supplementary Files}

This is a list of supplementary files associated with this preprint. Click to download.

- Supplementaryfile1.pdf

- Supplementaryfile2.pdf 